\title{
Regulierung und Haftung von Ratingagenturen - effizienteres Recht auf europäischer Ebene?
}

\author{
Thomas M.J. Möllers, Christine Wecker
}

Ratingagenturen gelten als ein Mitverursacher der Finanzkrise. In den USA und Europa wurden inzwischen zahlreiche Gesetze eingeführt, um Ratingagenturen besser zu überwachen. Es ist allerdings unbestritten, dass diese Regularien noch nicht ausreichen, um die während der Finanzkrise zutage getretenen Probleme zu lösen. Die Abhandlung bewertet den neuen Vorschlag der Kommission zur Änderung der Rating-VO, der etwa zwei obligatorische Ratings und ein Haftungsregime einführt. Will man die Rechtsdurchsetzung effektiv gestalten, bedarf es allerdings weiterer Maßnahmen: So könnte der gesetzliche Zwang zu einem (zweiten) europäischen Rating die oligopolistische Struktur des Marktes durchbrechen und kleinen europäischen Agenturen eine entscheidende Starthilfe geben. Ein Haftungssystem kann den Anreiz zur Einführung einer Selbstkontrolle nur dann steigern, wenn dieses auch abschreckt: Im Einzelnen müssen die Vorschläge des Gesetzgebers noch weiter konkretisiert werden, damit ein unrichtiges Rating, wie etwa bei Lehman-Brothers, tatsächlich einen Schadensersatzanspruch begründet. Dazu gehören die Verpflichtung zum unverzüglichen Anpassen des Ratings, die Verhinderung eines vorschnellen Herunterstufens des Emittenten und die Einführung eines Rating-Outlooks.

\section{A. Einleitung}

Ratingagenturen bläst dieser Tage ein scharfer Wind ins Gesicht: Die Regulierungsbemühungen des europäischen Gesetzgebers sind fortgeschritten, aktuell wird gar die Einführung eines Haftungsregimes diskutiert. Unternehmen stellten erstmals öffentlich die oligopolistische Marktstruktur in Frage und kritisierten die Preisbildung der Ratings mit scharfen Worten. ${ }^{1}$ Selbst unter den Ratingagenturen zeigen sich Unstimmigkeiten, etwa als Fitch den Konkurrenten Standard \& Poor's $(S \& P)$ im Rahmen eines Hypotheken-Bonds-Deal wegen vermeintlich zu positiver Bewertungen angriff. ${ }^{2}$ Schlagzeilen um Ratingpannen ${ }^{3}$, umstrittene

1 "Deutsche Großkonzerne wehren sich gegen Preiserhöhungen von S\&P«, reuters v. 23. April 2012, http://de.reuters.com/article/economicsNews/idDEBEE83M09R20120423.

$2 \quad$ Fitch in FAZ v. 4. April 2012, S. 19. 
Herabstufungen der Kreditwürdigkeit ${ }^{4}$ und die noch anhängige Klage ${ }^{5}$ eines geschädigten Anlegers gegen $S \& P$ heizen die Diskussion um die Regulierung von Ratingagenturen weiter an. Im Folgenden werden zunächst vor dem Hintergrund der alten Gesetzeslage (I.) die bisherigen Regulierungsbemühungen (II.) dargestellt. Anschließend werden bestehende Regelungslücken (III.), insbesondere die geplante Einführung eines Haftungsregimes (IV.), diskutiert.

\section{Gesetzeslage vor 2009}

Lange Zeit gab es im europäischen Recht keine umfassende Regelung zur Überwachung von Ratingagenturen. Das frühere System beruhte auf dem Prinzip der Selbstregulierung nach den Regelungen des IOSCO-Kodex ${ }^{6}$ und einem Anerkennungsverfahren im Rahmen der Eigenkapitalrichtlinie (Capital Requirements Directive $)^{7}$. Obwohl in den USA schon seit 1975 ein Zulassungsverfahren fur Ratingagenturen existierte, erachtete die Europäische Union die geltenden Standards als ausreichend: ${ }^{8}$ Unter anderem wurde angeführt, dass sich der Markt selbst reguliere, da Ratingagenturen ausschließlich von ihrer Reputation abhängig sind, ein Zulassungsverfahren den Markt abschotten und eine Art Mitverantwortung des Staates für die veröffentlichten Ratingurteile begründen würde. ${ }^{9}$

Drei elementare Instrumentarien existieren vorrangig, welche die Funktionsweise des Kapitalmarkts sicherstellen: Vermeidung von Interessenskonflikten,

»Rating-Panne empört Frankreich«, spiegelonline v. 11. November 2011, http://www. spiegel.de/wirtschaft/soziales/standard-poor-s-rating-panne-empoert-frank-reich-a-7971 16 html.

4 „United States loses prized AAA credit rating vom S\&P«, reuters v. 6. August 2011, http://www.reuters.com/article/2011/08/06/us-usa-debt-downgrade-idUSTRE7746VF 20110806.

5 "Klage gegen Ratingagentur zugelassen«, Handelsblatt v. 28. November 2011, http:/ www.handelsblatt.com/unternehmen/banken/nach-lehman-pleite-klage-gegen-ratingagentur-zugelassen/5895788.html.

6 Code of Conduct Fundamentals for Credit Rating Agencies (Verhaltenskodex) der IOSCO (International Organization of Securities Commissions), http://www.eesc.europa. eu/self-and-coregulation/documents/codes/private/065-private-act.pdf. Richtlinie 2006/48/EG v. 14. Juni 2006, ABI.EU Nr. L 177, 1 und Richtlinie 2006/49/EG v. 14. Juni 2006, ABl. Nr. L 177, 201.

8 Siehe Mitteilung der Kommission über Rating-Agenturen v. 11. März 2006, AB1. Nr. C 59, 2, 6: »Der Aufforderung des Europäischen Parlaments folgend hat sich die Kommission sehr eingehend mit der Frage befasst, ob in Bezug auf die Tätigkeit von RatingAgenturen neue Legislativvorschläge erforderlich sind und gelangte zu dem Schluss, dass dies derzeit nicht der Fall ist."

9 Zum Streit s. etwa Partnoy, 77 Wash.U.L.Q. (1999), 619, 627; Schwarcz, 1 U.Ill.L.Rev. (2002), 1; Fleischer, Gutachten F 64. DJT, 2002, F 134. 
Informationspflichten und Überwachung. ${ }^{10}$ Diese Instrumentarien waren Ratingagenturen gegenüber bisher aber nicht hinreichend ausgestaltet.

\section{Interessenkonflikte}

Seit den 1970er Jahren gilt das sogenannte »Issuer-Pays-Modell«, wonach der Emittent für die Erstellung des Ratings zahlt. ${ }^{11}$ Dies hat zur Folge, dass Ratingagenturen nicht frei von Interessenkonflikten und unabhängig agieren, weil sie regelmäßig von demjenigen Unternehmen ein Rating erstellen, von welchem sie beauftragt werden (sog. Auftragsrating oder »solicited rating «). ${ }^{12}$ Bei der Auswahl der Ratingagentur dürften Unternehmen bestrebt sein, eine höchstmögliche Bonitätsstufe zu erreichen. Dass diese Befürchtung nicht unbegründet ist, zeigte sich jüngst, als Fitch den Konkurrenten $S \& P$ öffentlich wegen vermeintlich zu positiver Bewertungen kritisierte. ${ }^{13}$ Verschärfend kommt hinzu, dass häufig zuvor das Unternehmen beraten wurde, nach welchen Kriterien ein Finanzprodukt strukturiert werden muss, um eine bestimme Note zu erreichen. Als besonders verheerend erwiesen sich in der Finanzkrise die sogenannten strukturierten Finanzprodukte wie die »Collateralised Debt Obligations« (CDO). Obwohl diese gegenüber anderen Finanzprodukten besonders gefährlich und risikoreich sind, wurden sie von den Ratingagenturen mit Bestnoten für höchste Ausfallsicherheit bewertet. $^{14}$

\section{Fehlende Information}

Der wirtschaftliche Hintergrund der Informationspflichten ist die Annahme von Kapitalmarkteffizienz. Diese Annahme beinhaltet, dass öffentliche Informationen unverzüglich in den Markt »eingespeist « werden, sobald sie dem Anlegerpublikum vorliegen. ${ }^{15}$ Die Behavorial-Finance-Forschung verdeutlicht, dass der

10 Möllers, 4 C.M.L.J. 477 f. (2009); ders., JZ 2009, 861 f.

11 Haar, ZBB 2010, 185, 187.

12 Zur Abgrenzung vom solicited zum unsolicited rating siehe Blaurock, ZGR 2007, 603, $604 \mathrm{ff}$.

13 "Obwohl wir nach einer Einschätzung der Transaktion gefragt wurden, erhielt Fitch wegen des konservativeren Bewertungsansatzes schließlich nicht den Auftrag für das Rating.«, so Fitch (Fn.2), S. 19.

14 Möllers (Fn. 10), 862, 867 f.

15 Bzgl. Informationseffizienz im Kapitalmarkt und der Efficient Capital Markets Hypothesis (ECMH), vgl. Fama 25 J.Fin. (1970), 83; ders. 46 J.Fin. (1991), 1575, 1576; Fischel, 74 Cornell. L. Rev. (1989), 907; Brealey/Myers, Principles of Corporate Finance, 7th ed., Boston 2003, S. 347 ff.; Elton/Gruber/Brown/Goetzmann, Modern Portfolio Theory and Investment, 6th ed. 2003, S. $402 \mathrm{ff}$. 
»homo oeconomicus«, der Informationen rational verarbeitet, ein selten anzutreffender Idealtypus ist: die Börsen überreagieren in Wellen, weil sie das von Emotionen getriebene Verhalten ihrer Marktteilnehmer abbilden. ${ }^{16}$

Die Finanzkrise hat gezeigt, dass die von den Agenturen verwendeten Informationen und damit die eigentlichen Ratings über das Unternehmen nicht vertrauenswürdig sind: Subprime-Produkte erhielten die höchsten Ratings, womit die großen Risiken dieser Instrumente klar unterbewertet wurden. Zudem versagten die Ratingagenturen, als es darum ging, die Ratings den sich verschlechternden Marktbedingungen unverzüglich anzupassen. ${ }^{17}$

\section{Oligopolistische Struktur des Ratingmarktes}

Bis heute dominieren weltweit drei Ratingagenturen den Markt; $S \& P$, Moody's und Fitch. ${ }^{18}$ Diese Marktstruktur ist darauf zurückzuführen, dass Unternehmen Ratingagenturen aufgrund ihrer Reputation auswählen. Die Zuverlässigkeit der Ratings beurteilt sich nach einer langjährigen und genau dokumentierten Historie, dem sog. track record. Dieser zeigt, ob eine Agentur über einen längeren Zeitraum mit ihren Ratings hinreichend gute Einschätzungen geliefert hat. ${ }^{19}$ Diese oligopolistische Struktur des Ratingmarkts schafft Abhängigkeiten und gleichzeitig Marktzugangsbarrieren ${ }^{20}$ für kleinere und mittlere Ratingagenturen, die sich zu etablieren versuchen.

16 Wie beispielsweise »lemmings«, die blind den Empfehlungen eines sog. Finanz-Gurus folgen. Vgl. allgemein für Behavioral Finance bspw. Thaler. Advances in Behavioral Finances Vol. I, Princeton 1993; ders. Advances in Behavioral Finances Vol. II, 2005; Goldberg/von Nitzsch, Behavioral Finance, 4. Aufl. 2004; Fleischer, in: FS Immenga, 2004, S. $575 \mathrm{ff}$.

17 Europäische Kommission, Vorschlag für eine Verordnung über Ratingagenturen v. 12. November 2008, KOM (2008) 704, 2, http://ec.europa.eu/internal_market/securities/ docs/agencies/proposal_de.pdf; ausführlich Möllers (Fn. 10), 861 ff.

$18 S \& P$ und Moody's haben gemeinsam $80 \%$ Marktanteil, die britische Ratingagentur Fitch weitere $15 \%$, vgl. Hill, Regulating the Rating Agencies, 82 Wash.U.L.Q. (2004), 43, 59 f.; Blaurock (Fn. 12), 606.

19 Zentraler Kreditausschuss, Stellungnahme zur Tätigkeit von Rating-Agenturen und ihrer möglichen Regulierung v. 14. August 2003, S. 5 ,

http://www.zka-online.de/uploads/media/030815_ZKAStn_Rating-Agenturen.pdf. Neuen Anbietern bleiben deshalb nur Marktnischen; Strunz-Happe, WM 2004, 115, 120.

Vgl. Lerch, BKR 2010, 402, 403; Thörle, BLJ 2011, 14. 
II. Europäische Regulierungsbemühungen im Zuge der Finanzkrise

1. Rating-VO Nr. $1060 / 2009^{21}$

Das Platzen der Subprime-Blase führte zu einem Umdenken in der europäischen Finanzmarktpolitik: Das Versagen der Ratingagenturen »lässt sich am besten durch Maßnahmen in den Bereichen Interessenkonflikte, Ratingqualität, Transparenz und (...) Beaufsichtigung der Tätigkeit von Ratingagenturen korrigieren ${ }^{22}$. Mit Erlass der Rating-VO im November 2009 erteilte die EU damit dem "Prinzip der Selbstregulierung ${ }^{23}$ eine Absage.

Kernstück der Rating-VO ist das Registrierungsverfahren mit der damit einhergehenden Registrierungspflicht in Art. 14 Rating-VO. Der Antrag der Ratingagentur war beim CESR (Ausschuss der Europäischen Wertpapierregulierungsbehörden) einzureichen ${ }^{24}$, der entsprechend seiner Beratungsfunktion Leitlinien ${ }^{25}$ und Empfehlungen ${ }^{26}$ abgab. Die Prüfung des Antrags oblag dabei letztlich den national zuständigen Behörden.

Mit dem Ausführungsgesetz zur Verordnung (EG) Nr. 1060/2009 (AusfG Rating-VO $)^{27}$ bestimmte der deutsche Gesetzgeber durch Änderung des § 17 WpHG die BaFin zur zuständigen Behörde im Sinne der Rating-VO und führte in $\S 39 \mathrm{WpHG}$ einen umfangreichen Ordnungswidrigkeitenkatalog ein.

2. ESMA-VO Nr. 1095/2010 und Änderungs-VO Nr. 513/2011

Bereits im de Larosière-Bericht ${ }^{28}$ war ein klares Mandat und eine neue Struktur zur Verstärkung der Wirksamkeit der Aufsicht gefordert worden. Da die materielle Prüfung der Zulassungsvoraussetzungen sowie aufsichtsrechtliche Maßnahmen weiterhin den nationalen Behörden oblagen, wurde die CESR nur bei Konflikten zwischen den zuständigen Behörden eingeschaltet. ${ }^{29}$ Der organisatorische Abstimmungsbedarf war damit erheblich. ${ }^{30}$ Im Zuge der ESMA-VO ${ }^{31}$ wurde die

21 Verordnung (EG) Nr. 1060/2009 v. 16. November 2009, ABl. Nr. L 302, 1 (Rating-VO); Möllers (Fn. 10), 870; ders., NZG 2010, 285, 288.

22 Erwägungsgrund 10 Rating-VO (Fn. 21).

23 Deipenbrock, RIW 2010, 612, 613.

24 Art. 15 Rating-VO (Fn. 21).

25 Vgl. CESR (ESMA), CESR Annual Report 2010, S. 42 ff.

26 Vgl. Hupka, WM 2009, 1351; Deipenbrock, WM 2011, 1829, 1830.

27 Ausführungsgesetz v. 14. Juni 2010 zur Verordnung (EG) Nr. 1060/2009 über Ratingagenturen, BGBI. I Nr. 32, 768.

28 Larosière Group, Report on Financial Supervision in the EU v. 25. Februar 2009, S. 46 f., http://ec.europa.eu/internal_market/finances/docs/de_larosiere_report_de.pdf.

29 Art. 24 Abs. 3 UA 2 Rating-VO (Fn. 21).

30 Vgl. im Einzelnen Möllers (Fn. 10), 861, 870; ders. (Fn. 21), 288. 
ESMA (Europäische Wertpapier- und Marktaufsichtsbehörde) am 1.1.2011 als Nachfolgeorganisation der CESR errichtet. Die Änderungs- $\mathrm{VO}^{32}$ übertrug ihr schließlich die ausschließliche Zuständigkeit für die Registrierung und Beaufsichtigung der Ratingagenturen. ${ }^{33}$ Damit wurde endlich das »komplexe Kooperations- und Koordinierungsregime zwischen CESR, den Herkunftslandbehörden und den übrigen zuständigen Behörden ${ }^{34}$ zugunsten einer zentralisierten europäischen Zuständigkeit geändert.

\section{B. Aktuelle Rechtslage \\ I. Vermeidung von Interessenkonflikten}

Art. 6 und 7 Rating-VO regeln den Umgang mit Interessenkonflikten und skizzieren grobe Zielvorgaben wie beispielsweise die Vermeidung von Einflüssen auf das Rating durch Geschäftsbeziehungen der Ratingagentur. Anhang I der Rating-VO konkretisiert diese Vorgaben umfassend in organisatorischer (Abschnitt A), operationeller (Abschnitt B) und personeller Hinsicht (Abschnitt C).

1. Beratungsleistungen

Die im Vorfeld erbrachten Beratungsleistungen der Ratingagenturen erzeugen ein großes Potential für Interessenkonflikte, wenn Finanzprodukte des Emittenten von der Ratingagentur im Hinblick auf eine möglichst hohe Bonitätsnote gestaltet werden. Dieser Problematik hat sich die Rating-VO angenommen und derartige Beratungsleistungen für das bewertete Unternehmen untersagt. ${ }^{35} \mathrm{Ne}-$ bendienstleistungen wie »Marktprognosen, Einschätzungen der wirtschaftlichen Entwicklung, Preisanalysen und andere Analysen allgemeiner Daten« sind ausdrücklich zugelassen, jedoch in den Abschlussberichten der Ratingagentur offenzulegen. ${ }^{36}$

31 Verordnung (EU) Nr. 1095/2010 v. 24.November 2010 zur Errichtung einer Europäischen Aufsichtsbehörde (ESMA-VO), ABl. Nr. L 331, 84.

32 Verordnung (EU) Nr. 513/2011 v. 11. Mai 2011 zur Änderung der Verordnung (EG) Nr. 1060/2009 über Ratingagenturen (Änderungs-VO), ABl. Nr. L 145, 30.

33 Art. 40a Änderungs-VO (Fn. 32).

34 Deipenbrock (Fn. 26), 1831; die dezentrale Zuständigkeit war mit deutlichen Worten kritisiert worden, so etwa Möllers (Fn. 10), 869 f.; Deipenbrock (Fn. 23), 617.

35 Anhang I Abschnitt B Abs. 4 Rating-VO (Fn. 21).

36 Anhang I Abschnitt B Abs. 2 und 3 Rating-VO (Fn. 21). 
Die Verordnung vermeidet die Adressierung des Kernproblems des Interessenkonflikts, namentlich des »Issuer-Pays«-Modells. Insofern verhindern die neuen Vorschriften die Interessenkonflikte nicht, sondern reduzieren sie nur. Weiterführend wäre es deshalb, wenn sich künftig die Ratings ohne Auftrag (»unsolicited ratings «) als Gattung durchsetzen würden, die von anderen Marktteilnehmern abgefragt und auch käuflich erworben werden könnten. Bisher existiert ein Markt für »unsolicited ratings« weitgehend nur für die Folgeratings nach einer Beauftragung durch ein Unternehmen, aber am Markt scheint inzwischen der Wettbewerb zuzunehmen. Die Egan-Jones Rating Company ${ }^{37}$ bietet etwa gar keine Auftragsratings mehr an, sondern wirbt damit, keinen Interessenkonflikten zu unterliegen. Denkbar wäre zudem, dass die Aufsichtsbehörden die Ratingagenturen zur Erstellung eines Ratings beauftragen. Im Kapitalmarktrecht ist eine solche Beauftragung etwa bei der Kontrolle von Wertpapierunternehmen durch Prüfer bekannt. Dort prüfen Wirtschaftsprüfer im Auftrag der Bundesanstalt für Finanzdienstleistungsaufsicht (BaFin), ob die Wertpapierdienstleistungsunternehmen ihre Aufklärungs- und Beratungspflichten eingehalten haben. ${ }^{38}$ Auch in den USA war die Einschaltung eines Intermediärs zwischenzeitlich diskutiert worden. ${ }^{39}$ Die SEC hat hierzu eine Machbarkeitsstudie in Auftrag gegeben. ${ }^{40}$

Der Ende 2011 von der ESMA vorgelegte Entwurf ${ }^{41}$ für verpflichtende Verhaltensstandards für Ratingagenturen (»Regulatory Technical Standards«, RTS) soll die Transparenz des Registrierungsverfahrens sowie der laufenden Ratingtätigkeiten weiter verbessern. Auch eine Abschaffung des Issuer-Pays-Modells wird darin erstmals eingehend diskutiert ${ }^{42}$, im Vorschlag der EU-Kommission

37 http://www.egan-jones.com/, deren Geschäft in den Jahren 2007 - 2009 um 30\% gewachsen sei, http://www.handelsblatt.com/unternehmen/banken/ratingagenturen-kleineratinghaeuser-hoffen-auf-marktanteile/3291592.html; Möllers (Fn. 10), 865.

38 Möllers, ZJS 2009, 227, 231.

39 Bächstädt, KRP $4 / 2010$, S. 24, http://www.ratingrisk.de/Baechstaedt_Fin Ratingagenturen.PDF; Vorschlag von Senator Franken, http://www.dsnews.com/articles/ sen-franken-and-others-address-rating-agency-reform-2011-09-01.

40 Securization Update von Kramer Levin Naftalis \& Frankel LLP, Jan. 2012, S. 8, http://www.kramerlevin.com/files/Publication/706e8e86-d4a2-4b69-8665-0b0bbbb3c9f5/ Presentation/PublicationAttachment/cf152c29-0b87-4dd6-840c-0f0fa0f94073/Securitization $\% 20$ Update $\% 20$ January $\% 202012$.pdf.

41 Final Report, ESMA/2011/461 v. 22. Dezember 2011, http://www.esma.europa.eu/ system/files/2011_461.pdf.

42 Bremer, NZG 2011, 1181. 
für eine neue Verordnung wird aber trotz der Einräumung von Interessenkonflikten nach wie vor an diesem Modell festgehalten. ${ }^{43}$

\section{Transparenz und Offenlegungspflichten}

Neben den Regelungen zur Vermeidung von Interessenkonflikten wurden den Ratingagenturen durch die Rating-VO umfassende Informations- und Offenlegungspflichten auferlegt. ${ }^{44}$ Art. 8 und 10 der Rating-VO sowie Art. 11 in Verbindung mit Anhang I Abschnitt E konkretisieren die Offenlegungspflichten im Hinblick auf Methoden, Modelle und grundlegende Annahmen, welche die Ratingagenturen ihren Bewertungen zugrunde legen. Auch über die historischen Ausfallquoten ihrer Ratingkategorien haben die Agenturen alle 6 Monate Rechenschaft abzulegen. ${ }^{45}$ Ferner müssen Ratingagenturen jährlich einen Transparenzbericht veröffentlichen, in dem unter anderem die internen Kontrollmechanismen zur Qualitätssicherung, die Methoden- oder Modellbewertungen sowie die Rotationspolitik der Analysten offengelegt werden. ${ }^{46}$ Mitgliedstaaten und Europaparlament einigten sich auf Basis des Vorschlags der Kommission KOM (2011) 747 nun zudem darauf, dass die Veröffentlichung von Länderratings nur noch jährlich an drei zuvor festgelegten Terminen und außerhalb der Börsenöffnungszeiten erlaubt sei, um dem viel diskutieren Veröffentlichungszeitpunkt von Ratings die Brisanz zu nehmen. ${ }^{47}$

C. Weitere Forderungen de lege ferenda: Reduzierung der Abhängigkeit der Finanzmarktteilnehmer von Ratings der Ratingagenturen

Binnenmarktkommissar Michel Barnier gab als Ziel der Regulierungsbemühungen an, »den übermäßigen Rückgriff auf Ratings zu verringern und gleichzeitig die Ratingverfahren qualitativ zu verbessern ${ }^{48}$. Ratings sollten transparenter gestaltet werden und die Agenturen haftbar gemacht werden. Auch die Verbesserung des Wettbewerbs würde angestrebt. Erste wichtige Schritte wurden bereits

43 Europäische Kommission, Vorschlag für eine Verordnung zur Änderung der RatingVerordnung (EG) Nr. 1060/2009 v. 15. November 2011, KOM (2011) 747, 9, http://ec.europa.eu/internal_market/securities/docs/agencies/COM_2011_747_de.pdf.

44 Hierzu ausführlich Haar (Fn. 11), $188 \mathrm{ff}$.

45 Cortez/Schön, Kreditwesen 2010, $226 \mathrm{f}$.

46 Art. 12 in Verbindung mit Abschnitt E Teil III Rating-VO (Fn. 21).

47 Pressemitteilung des Europäischen Parlaments v. 28. November 2012, http://www.europarl.europa.eu/news/de/pressroom/content $/ 20121127$ IPR56742/html/Dea 1-on-stricter-EU-rules-for-sovereign-debt-ratings.

48 EU-Kommission, Pressemitteilung vom 15. November 2011, EuZW 2011, 931. 
verwirklicht, weitere Maßnahmen befinden sich in der Umsetzung oder Diskussion.

\section{Aufbau einer europäischen Ratingagentur}

Das Modell einer europäischen Ratingagentur ist nicht neu ${ }^{49}$ und wird seit langem kontrovers diskutiert ${ }^{50}$. Ziel sei es, das bestehende Oligopol zu durchbrechen und ein Gegengewicht zur anglo-amerikanischen Dominanz im Ratingwesen zu schaffen. ${ }^{51}$ Fraglich ist jedoch, wie der Markt auf eine europäische Ratingagentur ohne Reputation reagiert. Dieser Aspekt verschärft auch die Diskussion über eine hoheitlich getragene europäische Ratingagentur:

Neben der Förderung von privatrechtlich ausgestalteten Ratingagenturen wurde die Errichtung einer von der EU-Kommission, Euro-Finanzministern und der EZB getragenen Ratingagentur nach dem Vorbild von Stiftung Warentest diskutiert $^{52}$, die ausschließlich Staatspapiere bewerten würde. Eine hoheitlich getragene Ratingagentur könnte jedoch die so vehement geforderte Unabhängigkeit bezüglich Länderratings nur schwer gewährleisten. Fraglich ist ferner, wie eine solche Institution öffentlichem und politischen Druck begegnen würde. ${ }^{53}$ Auch im Bundestag wurde bereits angemerkt, dass nur eine vom Staat unabhängige Ratingagentur Marktakzeptanz erringen könne. ${ }^{54}$

Zweifelsohne würde eine etablierte europäische Ratingagentur den Wettbewerb verbessern. Die Frage der Unabhängigkeit ist letztlich aber auch eine Frage der Perspektive: Bei Ratings durch eine europäische Agentur dürften sich seitens der USA und anderer Finanzmarktteilnehmer die gleichen Vorbehalte ergeben, wie die Europäer dieser Tage gegen die Bewertung durch die angelsächsischen Agenturen hegen. Mit einer Orientierung der europäischen Ratingagentur an der EZB wäre somit nichts gewonnen. Ob europäische Ratings angesichts der immer stärker homogenisierten Aufsichtsvorschriften in den USA und Europa qualitativ besser ausfielen, darf zudem bezweifelt werden. ${ }^{55}$ Folglich hat die EU-

49 Erfolglose Vorstöße wurden bereits 1980 und 2004 unternommen, vgl. Breuer, WM 1991, 1109; Red. EuZW 2004, 198.

50 So z.B. für eine europäische Ratingagentur: Bundeskanzlerin Merkel, Stellungnahme des EWSA v. 21. Oktober 2010, ABI. Nr. C 51, 18; CDU-Europaabgeordneter Langen, in: Friedrich, VW 2010, 1066; Europagruppen-Präsident Junker für ein von der EZB aufgebautes, unabhängiges Instrument des Länder-Ranking für Euro-Staaten, in: Friedrich, VW 2010, 427; kritisch etwa Oechsler, ZRP 2011, 191.

51 Gerke, BB 2011, 1.

52 Berschens/Cünnen/Landgraf/Osman, "Was eine neue Ratingagentur für Europa bringt», Handelsblatt v. 7. Mai 2010, S. 46; Thörle (Fn. 20), 18.

53 Wendt/Lampert, DVBl. 2010, 1001, 1007.

54 BT-Drucks. 17/7638, 3.

55 So auch Gerke (Fn. 51), 1; Lansch, VW 2010, 691. 
Kommission zuletzt Abstand von der Idee der Schaffung einer »Europäischen Ratingagentur « genommen. ${ }^{56}$

Im Frühjahr 2012 sollte die von der Unternehmensberatung Roland Berger als Stiftung konzipierte europäische Ratingagentur an den Start gehen. ${ }^{57}$ Zuerst werde die Abgabe von Länderratings im Vordergrund stehen, später sollen auch Unternehmens- und Bankenratings folgen. Roland Berger verfolgte dabei ehrgeizige Ziele. ${ }^{58}$ Die Konkurrenz ist groß: weltweit gibt es je nach Quelle 70 bis 150 Ratingagenturen, ${ }^{59}$ viele kleinere Ratingagenturen spezialisieren sich aber nur regional oder in Marktnischen. ${ }^{60}$

Es wird sich zeigen, inwieweit Investoren und Emittenten bereit sind, auf einen neuen Anbieter ohne langjährige Reputation zurückzugreifen. Erst drohte das Projekt schon vor Beginn wegen Finanzierungsproblemen zu scheitern ${ }^{61}$, zuletzt wurde ein neuer Anlauf für das Jahr 2013 angekündigt. ${ }^{62}$ Sollte Roland Berger das Vertrauen der Investoren gewinnen können, so muss sich künftig beweisen, ob das Label eines »europäischen Ratings « einen besonderen Qualitätsund Informationswert gegenüber US-Ratings verspricht und vom Markt als solches akzeptiert wird. ${ }^{63}$ Zuletzt erlebte die Idee der Errichtung einer unabhängigen europäischen Ratingagentur ein überraschendes Comeback, als sich im November 2012 Mitgliedstaaten und Europaparlament auf künftig schärfere Regelungen basierend auf dem Vorschlag der Kommission KOM (2011) 747 einigten. Die EU wolle die Gründung einer unabhängigen, nicht gewerbsmäßigen Ratingstiftung bis 2016 prüfen. ${ }^{64}$

56 Vgl. Europäische Kommission, VO-Vorschlag (Fn. 43), 13: „Ziel dieses Vorschlags ist nicht die Schaffung einer Europäischen Ratingagentur. (...) Die Folgenabschätzung ergab, dass (...) es schwierig wäre, Bedenken in Bezug auf Interessenkonflikte und ihre Glaubwürdigkeit zu zerstreuen, insbesondere wenn eine solche Ratingagentur Länderratings abgeben würde.«

57 Freiberger, »Nessie taucht auf«, SZ v. 23. Januar 2012, http://www.sueddeutsche. $\mathrm{de} /$ wirtschaft/europaeische-ratingagentur-nessie-taucht-auf-1.1264476.

58 Bereits innerhalb von fünf Jahren wird in Europa ein Marktanteil von 25\% angestrebt, nach zehn Jahren $25 \%$ weltweit, Buchter/Storn, »Allein gegen die großen drei«, Die Zeit v. 8. März 2012, S. 21.

59 Buchter/Storn (Fn. 58), S. 21.

60 Creditreform Rating Agentur in Deutschland bspw. konzentriert sich auf mittelständische Unternehmen, http://www.creditreform-rating.de/Deutsch/Rating/1_Wir_ueber_uns/ index.js; ASSEKURATA Assekuranz Rating-Agentur bewertet vornehmlich Versicherungen und Krankenkassen, http://www.assekurata.de/content.php?baseID=15.

61 »Roland-Berger-Projekt vor dem Aus«, FAZ v. 16. April 2012, S. 11.

62 »Europäische Ratingagentur steht vor der Gründung«, weltonline v. 27. April 2012 , http://www.welt.de/wirtschaft/article106233121/Europaeische-Ratingagentur-steht-vorder-Gruendung.html.

63 Bankenverband, „Was nützt eine europäische Ratingagentur?«, http://www.bankenverband.de/themen/politik-gesellschaft/defacto/defacto-14/was-nuetzt-eine-europaeische-rating-agentur.

64 SZ, "Schärfere Regeln für Ratingagenturen« v. 29. November 2012, S. 21. 
II. Verpflichtung zu einem europäischen Rating ${ }^{65}$

1. Vorschlag der Kommission zur Beauftragung von zwei Ratings

Der Vorschlag der EU-Kommission vom 15.11.2011 $1^{66}$ zur Änderung der Verordnung (EG) Nr. 1060/2009 sieht vor, dass Emittenten, die ein Rating in Auftrag geben, zwei verschiedene, voneinander unabhängige Ratingagenturen mit der Abgabe von Ratings für dieselben strukturierten Finanzinstrumente beauftragen müssen. Mit dieser Maßnahme soll die Qualität der Ratings sichergestellt werden. Die ESMA hat bisher 29 Ratingagenturen zugelassen und zertifiziert. ${ }^{67}$ Zwar stehen den Interessenten damit auf dem Papier eine Vielzahl an Ratingagenturen zur Auswahl, diese Zahlen können aber nicht darüber hinwegtäuschen, dass 95\% des Ratingmarktes nach wie vor von $S \& P$, Moody's und Fitch dominiert werden. Daher kann der Vorschlag der Kommission zwar als erster Schritt in die richtige Richtung zur Steigerung der Unabhängigkeit der Ratings gewertet werden; zur Durchbrechung des Oligopols müssen aber weitere Maßnahmen erfolgen.

2. Verpflichtung zum Nachweis eines europäischen Ratings

So wäre etwa an ein zweites obligatorisches Rating von einer europäischen Ratingagentur zu denken, das als Vergleichsmaßstab dient und Vertrauen schaffen soll. ${ }^{68}$ Ein solches Rating könnte die Dominanz der angelsächsischen Agenturen aufbrechen und würde gleichzeitig die Etablierung der kleineren europäischen Agenturen erleichtern und vorantreiben.

Mit Blick auf die derzeitigen durch die ESMA zugelassenen Ratingagenturen müsste die Definition einer europäischen Ratingagentur aber präzise ausgestaltet werden. $S \& P$ wird derzeit durch drei ihrer Töchter in der Union vertreten, Moody's durch sechs und Fitch durch sieben. Damit sind mehr als die Hälfte der durch die ESMA anerkannten Ratingagenturen europäische "Ableger« der "groBen Drei«. ${ }^{69}$ Die Forderung nach einem zusätzlichen Rating durch eine von der ESMA zertifizierte Agentur allein ist deshalb noch nicht zielführend. In Indien

65 Hierzu bereits Möllers/Wecker, ZRP 2012, 106.

$66 \operatorname{KOM}(2011) 747$, S. 8 (Fn. 43).

67 In Deutschland: Euler Hermes Rating, Feri EuroRating Services $A G$, Creditreform Rating $A G, P S R$ Rating $\mathrm{GmbH}, \mathrm{GBB}$-Rating Gesellschaft für Bonitätsbeurteilung $\mathrm{mbH}, \mathrm{AS}$ SEKURATA Assekuranz Rating-Agentur GmbH, Fitch Deutschland GmbH, Moody's Deutschland GmbH; List of registered and certified CRA's v. 6. Januar 2012, http://www.esma.europa.eu/page/List-registered-and-certified-CRAs.

Vgl. List of registered and certified CRA's v. 6. Januar 2012. 
etwa wird der Ratingmarkt von drei hierzulande eher unbekannten Agenturen CRISIL, CARE und ICRA beherrscht. ${ }^{70}$ Bei genauerer Betrachtung der Anteilseigner fällt aber auf, dass Moody's mit $29 \%$ an ICRA beteiligt und $S \& P$ gar mit $52 \%$ Mehrheitsaktionär von CRISIL ist. ${ }^{71}$ Derartige Zahlen belegen mit aller Deutlichkeit den Einfluss der amerikanischen Agenturen auf dem indischen Ratingmarkt.

Um eine ähnliche »Unterwanderung" der europäischen Agenturen in Europa zu verhindern, ist deshalb ein zweites Rating durch eine Ratingagentur zu fordern, die mit mindestens $50 \%$ von europäischen Anteilseignern gehalten wird. Auf diese Weise könnte zum einen erreicht werden, dass die vermeintliche Beeinflussung von Bewertungen durch anglo-amerikanische Interessen verhindert wird. Andererseits sollte aber durch zwei Ratings von tatsächlich verschiedenen Agenturen eine möglichst hohe Qualitätsstufe erzielt werden können. Unter diesem Gesichtspunkt verstößt der Vorschlag auch nicht gegen das Allgemeine Übereinkommen über den Handel mit Dienstleistungen (General Agreement on Trade in Services (GATS) $)^{72}$. Das Rating im Sinne einer Finanzdienstleistung fällt gemäß Abs. 1 (a) (xvi) der Anlage ${ }^{73}$ ausdrücklich in den Anwendungsbereich des GATS. Gem. Art. XVI Abs. 1 GATS wird der freie Marktzugang ausländischer Unternehmen zu den Dienstleistungsmärkten der GATSMitgliedstaaten in der Form vorgeschrieben, dass den Dienstleistungserbringern aus anderen Mitgliedstaaten hinsichtlich des Marktzugangs eine nicht weniger günstige Behandlung zu gewähren ist, als die jeweils in der Liste vereinbarte. ${ }^{74}$ Für Deutschland gilt hier die Liste der Verpflichtungen der EU-Staaten. ${ }^{75}$ Mit der Schaffung eines zweiten obligatorischen europäischen Ratings würde sich aber der Marktzugang für die bisherigen Anbieter gerade nicht verändern. Nichteuropäischen Agenturen wäre der Zutritt zum europäischen Markt nicht verwehrt, da der Rückgriff auf die etablierten Ratingagenturen durch ein "freies" Rating nach wie vor möglich ist. Der Ratingmarkt würde sich lediglich für die europäischen Agenturen erweitern. Auch bleibt es Finanzinstituten weiterhin unbenommen, noch weitere zusätzliche Ratings einzuholen. Zudem sind gemäß des GATS nicht schlechterdings sämtliche, den Marktzugang ausländischer Dienstleister potentiell einschränkende Maßnahmen zu unterlassen: „Ungeachtet etwa-

70 The Economist, »Lettters from India« v. 17. März 2012, S. 68.

71 The Economist, "Lettters from India« v. 17. März 2012, S. 68; CARE dagegen wird mehrheitlich von indischen Banken gehalten: IDBI Bank Ltd. $(26 \%)$, Canara Bank $(23 \%)$ und State Bank of India (9\%), http://www.careratings.com/AboutCARERatings/ Shareholders.aspx.

72 BGBI. II, 1994, $1643 \mathrm{ff}$.

73 BGBl. II, 1994, $1660 \mathrm{ff}$.

74 Vgl. ausführlich Vahldiek, BKR 2003, $971 \mathrm{ff}$.

75 Bekanntmachung des Fünften Protokolls zum Allgemeinen Übereinkommen über den Handel mit Dienstleistungen, BGB1. II, 1999, 312 ff.; BGBl. II, 1994, 1521 ff. 
iger sonstiger Bestimmungen des Übereinkommens wird ein Mitglied nicht daran gehindert, aus Gründen seiner Aufsichtspflichten Maßnahmen einschließlich Maßnahmen zum Schutz von Investoren, Einlegern, (...) oder zur Sicherung der Integrität und Stabilität seines Finanzsystems zu treffen. « $^{76}$

Freilich könnte auch eine derartige gesetzliche Regelung nicht verhindern, dass die großen Drei ihre Marktpräsenz in Europa stärken, indem sie beispielsweise kleinere Agenturen aufkaufen. Eine Verdrängung von $S \& P$, Moody's und Fitch soll und kann aber selbstverständlich nicht das Ziel einer solchen Maßnahme sein. Vielmehr soll der Ratingmarkt für europäische Agenturen geöffnet und diesen durch die Ankurbelung des Wettbewerbs eine reelle Chance eröffnet werden. Hierfür könnte die Verpflichtung zum Nachweis eines europäischen Ratings der Türöffner sein. Als weiterer begrüßenswerter Nebeneffekt ist die Errichtung der viel diskutierten »einen « europäischen Ratingagentur hinfällig: die Unabhängigkeit des Ratings wird durch den Wettbewerb der europäischen Agenturen unter sich gewährleistet und ein Gegengewicht zu vermeintlich US-affinen Ratings wäre geschaffen.

\section{Reduzierung der gesetzlichen Anknüpfungstatbestände}

\section{CRD-IV-Richtlinie/Basel III}

Mit Umsetzung der Basel II Eigenkapitalrichtlinien ${ }^{77}$ ist die Bedeutung der Ratingagenturen im europäische Bankenaufsichtsrecht gewachsen. ${ }^{78}$ Durch die neuen Regelungen sollte die risikogerechtere Eigenkapitalunterlegung von Krediten ermöglicht werden. Basis für diese Risikogewichtung sind interne oder externe Ratings, anhand derer die Ausfallwahrscheinlichkeit der Kreditrückzahlung bemessen wird. ${ }^{79}$ Damit wurde die Mindestkapitalausstattung der Kreditinstitute von der Bonität ihrer Schuldner abhängig gemacht. Solche Ratings dürfen in Deutschland nur von anerkannten Ratingagenturen durchgeführt werden. ${ }^{80}$

76 Sog. "prudential carve-out«, Ziff. 2 a) der Anlage zu Finanzdienstleistungen, BGBl. II, 1994, 1660.

77 Richtlinie 2006/48/EG v. 14. Juni 2006, ABl. Nr. L 177, 1 und Richtlinie 2006/49/EG v. 14. Juni 2006, ABl. Nr. L 177, 201.

78 Haar (Fn. 10), 193; so z.B. Art. 80 1. Richtlinie 2006/48/EG: "Zur Bewertung der Kreditqualität können (...) die Ratings von Ratingagenturen (...) herangezogen werden.«.

79 Vgl. Becker, DB 2010, 941, 943.

$80 \S \S 41,52 \mathrm{f}$. SolvV, Verordnung über die angemessene Eigenmittelausstattung von Instituten, Institutsgruppen und Finanzholding-Gruppen (Solvabilitätsverordnung - SolvV) v. 14.Dezember 2006, zuletzt geändert durch Artikel 1 der Zweiten Verordnung zur weiteren Umsetzung der geänderten Bankenrichtlinie und der geänderten Kapitaladäquanzrichtlinie v. 26. Oktober 2011 (BGBl. I, 2011, 2103). 
Derartige gesetzliche Verweisungen schufen Abhängigkeiten der Aufsichtsbehörden von Bewertungen nichtstaatlicher Institutionen und hatten Asymmetrien bezüglich der Marktmechanismen zur Folge. ${ }^{81}$ Diese Problematik wurde mittlerweile erkannt und der Normgeber hat reagiert, um den gesetzlichen Rückgriff auf das Urteil von Ratingagenturen in Zukunft zu reduzieren. Der Basel-IIIAkkord sieht vor, dass die Anrechenbarkeit von Mitteln als Aktiva sowohl von Ratings als auch von weiteren qualitativen und quantitativen Kriterien bestimmt werden soll. Als geeignete Kriterien würden unter anderem Volumen, GeldBrief-Spanne, und Umsatz herangezogen werden. Je robuster dabei diese Kriterien werden, umso weniger Gewicht sollte auf externe Ratings und umso mehr Gewicht auf die zusätzlichen Kriterien gelegt werden. ${ }^{82}$ Indem weitere Messgrößen zur Liquiditätsbewertung angeboten werden, soll übermäßiges Vertrauen allein in externe Ratings vermieden werden. Gegenwärtig werden solche Kriterien noch vom Ausschuss entwickelt und geprüft. ${ }^{83}$

Die Umsetzung erfolgt in der Europäischen Union über Änderungen der Capital Requirements Directive (CRD) und soll ab 2013 schrittweise in Kraft treten: Die EU-Kommission hat im Juli 2011 hierzu Vorschläge vorgelegt, die früheren Eigenkapitalrichtlinien (2006/48/EG und 2006/49/EG) zu ersetzen. ${ }^{84}$ Das Gesetzespaket aus einer Verordnung zur Tätigkeit der Kreditinstitute und Wertpapierfirmen und einer Richtlinie über die Zulassung zum Einlagengeschäft soll den EU-Bankensektor stärken. ${ }^{85}$ Unter anderem wird vorgesehen, dass Banken ihre Anlageentscheidungen künftig nicht nur auf externe Ratings stützen können, sondern dass sie auch ein eigenes internes Bonitätsurteil erstellen müssen. ${ }^{86}$ Ferner wäre bei einer maßgeblichen Zahl an offenen Positionen in einem Portfolio ein internes Rating für dieses Portfolio zu erstellen. Mit diesen Maßnahmen soll

81 Lerch (Fn. 20), 402; Siekmann, Verw 43, 95, 105; Deipenbrock, WM 2005, 266 ff.; dies., WM 2006, $2237 \mathrm{f}$.

82 Basler Ausschuss für Bankenaufsicht, Basel III: Internationale Rahmenvereinbarung über Messung, Standards und Überwachung in Bezug auf das Liquiditätsrisiko, Dezember 2010, S. 11, http://www.bundesbank.de/download/bankenaufsicht/pdf/basel3_liquidity_ rules.de.pdf.

83 Basler Ausschuss für Bankenaufsicht, S. 11 (Fn. 80); Basel III - Leitfaden zu den neuen Eigenkapital- und Liquiditätsregeln für Banken 2011, S. 23, http://www.bundesbank.de/ download/bankenaufsicht/pdf/basel3 leitfaden.pdf.

84 Vorschlag der Kommission für eine Richtlinie über den Zugang zur Tätigkeit von Kreditinstituten und die Beaufsichtigung von Kreditinstituten und Wertpapierfirmen und zur Änderung der Richtlinie 2002/87/EG über die zusätzliche Beaufsichtigung der Kreditinstitute, Versicherungsunternehmen und Wertpapierfirmen eines Finanzkonglomerats v. 21. Juli 2011, KOM (2011) 453, http://www.bundesbank.de/download/bankenaufsicht/pdf/capital_requirements_directive_de.pdf.

85 So Binnenmarktkommissar Michel Barnier am 20. Juli 2011, http://ec.europa.eu/ deutschland/press/pr_releases/10102_de.htm.

$86 \mathrm{Vgl}$, Erwägungsgrund 51 und Art. 77(b) KOM (2011), 453 (Fn. 84). 
die Bedeutung externer Ratings für Kreditinstitute verringert werden. ${ }^{87}$ Instituten soll einerseits ein Anreiz gegeben werden, selbständige Bewertungsmodelle zu entwickeln. Andererseits kann aber immer noch auch auf standardisierte Ansätze, die sich auf externe Ratings stützen, zurückgegriffen werden. In erster Linie sollen Finanzunternehmen dazu angehalten werden, nicht blind auf externe Ratings zu vertrauen sondern sich mit Hilfe der internen Bewertung ein eigenes Urteil zu verschaffen. ${ }^{88}$ Damit wird zwar die Abhängigkeit von externen Ratings reduziert, das Prinzip des Basel-II-Akkords wird letzten Endes aber nur abgemildert $^{89}$, da die Finanzunternehmen lediglich dazu angehalten werden, »nicht übermäßig auf Ratings zurückgreifen ${ }^{90}$.

\section{Solvency II}

Auch auf dem europäischen Versicherungsmarkt gewannen Ratings mit der Einführung der Solvabilitätsvorschriften ${ }^{91}$ an Bedeutung. Ziel ist es, die Eigenmittelanforderungen für Versicherungsunternehmen zu einem risikoorientierten System der Finanzaufsicht weiterzuentwickeln und das interne Risikomanagement der Versicherer zu verbessern. ${ }^{92}$ Auch hier wird auf die Ermittlung der Ausfallwahrscheinlichkeit abgestellt. Solvency II lässt den Unternehmen aber die Wahl, mittels welcher Methode die Risikomessung durchgeführt wird. Dabei können interne Modelle im gleichen Maße zum Einsatz kommen wie externe Bewertungen, sofern sie von den Behörden anerkannt werden. Im Jahr 2010 haben von 2.520 befragten europäischen Versicherungsunternehmen 289 angegeben, dass sie an einer Implementierung eines internen Modells arbeiten. ${ }^{93}$ In Deutschland verwenden gegenwärtig etwa ein Drittel der Lebens- und Krankenversicherungen und ein Viertel des Schadens- und Unfallversicherungsbereichs durch die BaFin zugelassene interne Modelle. ${ }^{94}$

87 Weber, NJW 2012, 274, 275; »Neue Regeln für Europas Banken« v. 21. Juli 2011, http://www.euractiv.de/finanzen-und-wachstum/artikel/neue-bankenregeln-fr-europa005124.

88 Vgl, Erwägungsgrund 51 und S. 7, KOM (2011), 453 (Fn. 84).

89 Witte, WM 2011, 2253.

$90 \operatorname{KOM}(2011) 757$, S. 8 (Fn. 43).

91 Richtlinie 2009/138/EG v. 25. November 2009 betreffend die Aufnahme und Ausübung der Versicherungs- und der Rückversicherungstätigkeit (Solvabilität II), ABI. Nr. L 335. 1 .

92 Will/Sönnichsen, VW 2010, 53.

93 EIOPA Report on the fifth Quantitative Impact Study (QIS5) for Solvency II, EIOPATFQIS5-11/001, 14. März 2011, S. 106, https://eiopa.europa.eu/publications/reports/ index.html.

94 BaFin - Ergebnisse der fünften quantitativen Auswirkungsstudie zu Solvency II (QIS 5),

S. 22, http://www.bafin.de/cln_228/SharedDocs/Downloads/DE/Unternehmen/Versich- 
Die regulatorische Abhängigkeit von Ratingagenturen im Versicherungswesen ist insofern zwar nicht gesetzlich bedingt; es zeigt sich aber, dass nur große internationale Versicherungsunternehmen über solche Modelle verfügen. ${ }^{95} \mathrm{Da}$ mit werden besonders kleine und mittelgroße Dienstleister im Regelfall auf externe Ratings zurückgreifen. ${ }^{96}$ Sowohl Solvency II als auch Basel III fördern also den Einsatz interner Risikobewertungsmodelle, um die Abhängigkeit von externen Ratings zu verringern. Es steht aber zu befürchten, dass die Kreditinstitute im Hinblick auf interne Ratings mit den gleichen vorstehend aufgezeigten Schwierigkeiten zu kämpfen haben werden wie die Versicherungsunternehmen.

\section{Haftung}

I. Prognoseentscheidung und Komplexität des Ratings

Als jüngstes Instrumentarium wird auf europäischer Ebene ein Schadenersatzanspruch vorgeschlagen. Allerdings ist die konkrete Ausgestaltung nicht ganz einfach. Die Problematik der Haftung von Ratingagenturen gegenüber Anlegern ergibt sich aus der Natur des Ratings als Bonitätsurteil selbst. Ein Rating gibt eine Prognose über die zukünftige Zahlungsfähigkeit und Zahlungswilligkeit eines Schuldners ab. Zwar wird durch die standardisierte Faktenbasis der Eindruck einer objektiven Beurteilung erweckt; letzten Endes handelt es sich dennoch um eine Zukunftsprognose, die dem Ersteller einen erheblichen Beurteilungsspielraum belässt. ${ }^{97}$

Beim »solicited rating" beauftragt der Emittent selbst eine Agentur mit der Erstellung eines Ratings. Hierfür werden von den Analysten verschiedenste Informationen gesammelt und bewertet: quantitative Daten wie Umsatz, Cash Flow und Eigenkapitalquote werden ebenso berücksichtigt wie qualitative Informationen über die Unternehmenskultur, Mitarbeiterpotential oder Risikomanagement. ${ }^{98}$ Anhand dieser Daten wird ein Rating erstellt. Die Bonität spiegelt sich dabei in einer Notenskala von »AAA» (höchste Bonitätsstufe) bis $» \mathrm{D} «$ (In-

ererUndPensionfonds/Solvency_II/qis5/qis5 ergebnisse bericht bafin,templateld= raw,property=publicationFile.pdf/qis5_ergebnisse bericht_bafin.pdf; Die Zulassungsvoraussetzungen statuiert Art. 121 der Richtlinie 2009/138/EG (Fn. 89).

95 Kaserer, Solvency II und Basel III, Die Reform der europäischen Versicherungs- und Bankenregulierung und deren Auswirkungen auf die Unternehmensfinanzierung, Juni 2011 , S. 16, http://www.fpmi.de/tl_files/fpmi/downloads/de/Gutachten_im_Auftrag der_fpmi.pdf.

$96 \bar{A}$ Arneth/Sauka, Kreditwesen 2008, 796, 799, http://www.meag.com/reddot/downloads/ de/Solvency_II.pdf.

97 Blaurock (Fn. 12), 627.

98 Fey/Birle, Beck'sches Steuer- und Bilanzrechtslexikon, Rn. 8. 
solvenz) wieder. ${ }^{99}$ Neben der Schwierigkeit der Einschätzung einer zukünftigen Entwicklung unterliegt die Würdigung der quantitativen Kriterien einem erheblichen subjektiven Beurteilungsspielraum. Auch verfolgt jede Ratingagentur ein eigenes Urteilsschema, sodass den jeweiligen Kriterien durchaus unterschiedliches Gewicht zukommen kann. ${ }^{100}$ Nach Abschluss der Analyse wird dann das Rating dem bewerteten Unternehmen vorgelegt, sodass sich dieses dazu äußern kann, bevor es veröffentlicht wird. ${ }^{101}$

Um die Haftung für ein fehlerhaftes Rating zu begründen, muss man sich deshalb mit dem Rating als Prognoseentscheidung über eine zukünftige Ausfallwahrscheinlichkeit auseinandersetzen, aber auch die Komplexität des Ratings als solchen würdigen. Gerade weil ein Rating keine reine Tatsachenbehauptung ist, kann aus der bloßen Tatsache, dass sich ein Bonitätsurteil ex post als unzutreffend herausgestellt hat, nicht automatisch die Fehlerhaftigkeit des Ratings geschlossen werden. ${ }^{102}$

Das Problem der Haftung für Prognoseentscheidungen findet sich auch im Bereich der Anlageberatung wieder. ${ }^{103}$ Hier wird eine Haftung in der Regel nur dann ausgelöst, wenn die Prognosen des Anlageberaters auf falschen Angaben beruhen, also die Tatsachenbasis unrichtig ist. ${ }^{104}$ Ausnahmsweise soll eine Prognose aber auch dann eine Haftung nach sich ziehen, wenn sie aus ex-ante Sicht völlig unvertretbar ist - unabhängig davon, ob sie auf falschen tatsächlichen Angaben beruht oder nicht. ${ }^{105}$ Vergleichbares gilt für Finanzanalysen. ${ }^{106}$

\section{Die bisherige Rechtslage}

1. USA und Australien

Von 1980 bis ins Jahr 2010 waren die Ratingagenturen in den USA von der sog. "Expertenhaftung " (»expert liability ${ }^{107}$ ) ausgenommen. ${ }^{108}$ Mit Inkraftreten des Dodd-Frank Wall Street Reform and Consumer Protection Act wurde diese Ausnahme aufgehoben. ${ }^{109}$ Ratingagenturen unterliegen damit der Haftung, soweit sie

99 Fey/Birle (Fn. 98), Rn. 8.

100 Rieg, BC 2004, $57 \mathrm{f}$.

101 Fey/Birle (Fn. 98), Rn. 38.

102 Hennrichs, in: FS Hadding, 2004, S. 875, 881.

103 Ausführlich hierzu Reinelt, NJW 2009, 1, 5 ff.

104 Z.B. BGH, NJW 2007, 1874; BGH NJW 2008, 649.

105 Reinelt (Fn. 104), 6.

106 Vgl. Möllers/Lebherz, BKR 2007, 349, 351.

107 »expert liability« gem. $\S \S 7$ und 11 Securities Act of 1933 (SA).

108 Gesetzlich kodifizierter Haftungsausschluss durch Rule 436(g) SA.

109 § 939G Dodd-Frank Act, H.R. 4173. 
der Aufnahme ihrer Ratings in Emissionsprospekten und Anträgen auf Börsenzulassung zustimmen. Nach Items 1103(a)(9) und 1120 der Regulation AB muss nämlich für jede Emission von forderungsbesicherten Wertpapieren (assetbacked securities, ABS) das zugrunde liegende Rating sowie der Name der Ratingagentur offengelegt werden. ${ }^{110}$ Ratings stellen somit notwendige Bestandteile im Börsenzulassungsverfahren und für ABS-Emissionsangebote dar. Um der Expertenhaftung zu entgehen, verweigerten die NRSROs die Zustimmung zur Veröffentlichung ihrer Ratings. ${ }^{111}$ Infolgedessen brach der Markt für forderungsbesicherte Wertpapiere zusammen. Als Reaktion darauf erließ die SEC einen "non-action-letter « ${ }^{112}$, ein Nichtanwendungserlass für die bestehenden Offenlegungsregelungen der Regulation $\mathrm{AB}$. Die SEC sieht damit vom Vollzug der Offenlegungspflichten in den oben genannten Fällen ab, sodass ABS-Transaktionen ohne Rating möglich sind. ${ }^{113}$ Obwohl diese Empfehlung der SEC zuerst zeitlich befristet war, wurde sie kürzlich auf unbestimmte Zeit verlängert. ${ }^{114}$ Die haftungsrechtliche Situation in den USA bleibt somit trotz der gesetzlichen Regelung undurchsichtig und es bleibt auf eine klärende Stellungnahme der SEC zu warten. ${ }^{115}$

Lange Zeit standen Ratings in den USA als »Meinungsäußerung « unter dem Schutz des »First Amendments«, wodurch eine Haftung für »fehlerhafte« Ratings praktisch ausgeschlossen war. ${ }^{116}$ Diese Rechtsprechung wurde 2009 erstmals aufgegeben. ${ }^{117}$ Obwohl Ratingagenturen somit grundsätzlich haftbar gemacht wurden, liegt die Hürde für eine erfolgreiche Schadensersatzklage nach wie vor sehr hoch: der Kläger muss der Ratingagentur eine erhebliche Verletzung der Aufklärungspflicht oder Täuschung nachweisen. ${ }^{118}$ Bisher gab es in den

110 SEC, Response of the Office of Chief Counsel Division of Corporation Finance v. 23. November 2010, http://www.sec.gov/divisions/corpfin/cf-noaction/2010/ford072210$1120 . \mathrm{htm}$.

111 Harper, 68 Wash. \& Lee L. Rev. 1925, 1926, 1964 (Fall 2011).

112 Ford Motor Credit Company LLC, SEC No-Action Letter (July 22, 2010).

113 »Hey, S.E.C., That Escape Hatch Is Still Open«, The New York Times v. 5.3.2011, http://www.nytimes.com/2011/03/06/business/06gret.html.

114 Ford Motor Credit Company LLC, SEC No-Action Letter (November 23, 2010), http://www.sec.gov/divisions/corpfin/cf-noaction/2010/ford072210-1120.htm.

115 Vgl. Brody/Hanawalt, Dodd-Frank: Rating Agencies And The ABS Market, Law 360, 24. Januar 2011, http://www.bingham.com/ExternalObjects/Docs/Dodd-Frank\%20Rating \%20Agencies\%20And\%20The\%20ABS\%20Market_\%286247\%29.pdf; Letzler, Expert liability for rating agencies, 3. Oktober 2011, The National Law Journal, http://www. law.com/jsp/nlj/PubArticleNLJ.jsp?id=1202517569077\&slreturn=1.

116 Berger/Stemper, WM 2010, 2289.

117 Abu Dhabi Commercial Bank v. Morgan Stanley \& Co. Inc., 651 F. Supp. 2d 155 (S.D.N.Y. 2009).

118 "material misrepresentations or omissions«, Genesee County Employees Retirement System v. Thornburg Mortgage Securities Trust 2006-3, 2011 WL 5840482 (D.N.M.Nov. 12, 2011) (Thornburg), at 148, http://www.stblaw.com/google_file.cfm?TrackedFile 
USA noch keine erfolgreiche Klage gegen eine Ratingagentur. Dies könnte sich mit dem vor dem U.S. District Court for the District of New Mexico noch anhängigen Verfahren ${ }^{119}$ ändern: Das Gericht versagte den beklagten Ratingagenturen Moody's, S\&P und Fitch die Berufung auf den Schutz der Meinungsfreiheit und hielt sie für grundsätzlich haftbar. Weil jedoch der Nachweis einer Verletzung der Aufklärungspflicht oder Täuschung nur bezüglich $S \& P$ gelang, wurde die Klage gegen Fitch und Moody's einstweilen teilweise abgewiesen. Das Gericht gestattete dem Kläger jedoch, die Klageschrift dahingehend zu ergänzen. ${ }^{120}$ Der Ausgang dieses Verfahrens könnte richtungsweisend für die Haftungsfrage der Ratingagenturen in den USA werden und erstmalig zum Erfolg für geschädigte Anleger führen.

Erstmals wurde im November 2012 ein Verfahren in Australien zugunsten der geschädigten Anleger entschieden: $S \& P$ wurde von einem australischen Bundesgericht zu 20 Millionen Dollar Schadensersatz verurteilt. ${ }^{121}$ Zwölf australische Gemeinden hatten geklagt, weil sie in von $S \& P$ mit der Bestnote »AAA« bewertete Finanzprodukte investiert und in der Finanzkrise 90\% der etwa 16 Millionen investierten Dollar verloren hatten. Das Gericht attestierte der Ratingagentur hierbei eine »irreführende und betrügerische Handlungsweise ${ }^{122} . S \& P$ hat Berufung angekündigt.

\section{Deutschland}

Wird der Anleger geschädigt, weil er sich bei seiner Anlageentscheidung auf ein Bonitätsurteil verlassen hat, kommen nach nationalem Recht verschiedene haftungsbegründende Ansätze in Betracht, deren Durchsetzung sich aber bisher als wenig erfolgsversprechend erwies: Nimmt man einen stillschweigend geschlossenen Auskunftsvertrag zwischen Ratingagentur und Anleger an, so wird es im Regelfall am Rechtsbindungswillen der Ratingagentur fehlen. Ein Anspruch aus Vertrag mit Schutzwirkung zugunsten Dritter kreist um das Problem des hinreichend bestimmbaren Personenkreises. Bei der sog. »Expertenhaftung« nach

=4B46116604DBE3D896B179\&TrackedFolder=585C1D235281AED9B6A07D5F9F94 78AB5А90188899.

119 Genesee County Employees Retirement System v. Thornburg Mortgage Securities Trust 2006-3, 2011 WL 5840482 (Thornburg) (Fn. 118).

1202011 WL 5840482, 130, 148 (Thornburg) (Fn. 118).

121 "Standard \& Poor's hit with \$20m compensation for wonky ratings«, The Sydney Morning Herald v. 6. November 2012, http://www.smh.com.au/business/standard--poors-hitwith-20m-compensation-for-wonky-ratings-20121105-28u32.html.

122 "misleading and deceptive conduct ", Bathurst Regional Council v Local Government Financial Services Pty Ltd (No. 5) (2012) FCA 1200, Ziff. 53, http://www.lemonde. $\mathrm{fr} / \mathrm{mmpub} / \mathrm{edt} / \mathrm{doc} / 20121105 / 1785835$ _4e5b_summary_lgfs.pdf. 
$\S \S 280$ Abs. 1, 311 Abs. 3 S. 2, 241 Abs. 2 BGB ergeben sich Beweisschwierigkeiten, dass das der Ratingagentur entgegengebrachte besondere Vertrauen gerade erheblich für die Investitionsentscheidung war. Ansprüche aus $\S \S 823$ Abs. 1 und 2 BGB scheitern wiederum am Vermögensschaden beziehungsweise daran, dass die Rating-VO kein Schutzgesetz gem. $\S 823$ Abs. 2 BGB ist. Ein Anspruch wegen vorsätzlicher sittenwidriger Schädigung gem. $\S 826$ BGB kommt nur dann in Betracht, wenn der Nachweis gelingt, dass das Rating nicht der Information der Markteilnehmer dient, sondern vielmehr zur Schädigung des auf die Ratings vertrauenden Anlegers instrumentalisiert wurde. ${ }^{123}$

Im Juni 2010 reichte ein Anleger beim LG Frankfurt a. M. Klage gegen $S \& P$ wegen deren Lehman-Ratings ein. Die Schadensersatzforderung beläuft sich auf $30.000 €$. Der Kläger hatte Lehman-Zertifikate erworben, die von der Ratingagentur mit der Bonitätsstufe A+ beurteilt worden waren. Als die Bank im September 2008 zusammenbrach, wurden die Zertifikate für den Kläger wertlos. Der Geschädigte machte vor Gericht geltend, dass bei sorgfältiger Bewertung der Finanzinstrumente durch $S \& P$ die Kreditwürdigkeit von Lehman $»$-schon mangels hinreichendem freien Eigenkapital zur Unterlegung der Risiken - nicht wie abgegeben (als) »gut«, sondern (als) »unsicher« oder »ausfallgefährdet« hätte bewertet werden müssen ${ }^{124}$. Zudem sei trotz massiver Berichterstattung in der Fachpresse über die drohende Insolvenz von Lehman Brothers die nur leicht abgewertete Bonität von »A« durch $S \& P$ bis drei Tage vor der tatsächlichen Insolvenz beibehalten worden. Die Ratingagentur hafte für den entstandenen Schaden wegen der »Verletzung der Überwachungs- und Anpassungspflicht des Ratings« nach den Grundsätzen eines Vertrags mit Schutzwirkung zugunsten Dritter sowie - hilfsweise - gem. $\S 2$ Abs. 3a Nr. 5 o. 6 WpHG nach Maßgabe der $\S \S 31 \mathrm{ff}$. WpHG. ${ }^{125}$

Der Kläger scheiterte zunächst, da sich das Gericht für örtlich unzuständig erklärte. ${ }^{126}$ Im Berufungsverfahren folgte das OLG Frankfurt der Argumentation des Klägers und verwies das Urteil zurück an das LG Frankfurt a. M. ${ }^{127}$ Der Ausgang des Verfahrens bleibt abzuwarten.

123 Ausführlich Berger/Stemper (Fn. 116), 2292; Haar, NZG 2010, 1281 ff.; vgl. auch BGH DStR 2004, 1490 und BGH NJW 2004, 2664.

124 Klageschrift v. 1. Juni 2010, S. 6; www.kapitalmarktrecht-im-internet.eu.

125 Vgl. Klageschrift, S. 9 (Fn. 124).

126 LG Frankfurt v. 20. April 2011, 2/13 O 111/10.

127 OLG Frankfurt v. 28. November 2011, WM 2011, 2360. 
1. Pflichtverletzungen im Sinne des Art. 35a

Neu ist dagegen ein Vorschlag der Kommission ${ }^{128}$ erstmals ein konkretes Haftungssystem durch den Verordnungsvorschlag in Erwägung zu ziehen. So sollen Ratingagenturen nach Art. 35a bei vorsätzlichen oder grob fahrlässigen VerstöBen gegen die Verordnung haften, durch die einem Anleger, der sich auf ein Rating dieser Agentur verlassen hat, ein Schaden entstanden ist, wenn der betreffende Verstoß sich auf das Rating ausgewirkt hat.

Anhang III der Verordnung Nr. 1060/2009 soll dabei um einen umfassenden Pflichtenkatalog ergänzt werden. Ein haftungsbegründender Verstoß gegen die Verordnung läge demnach vor, wenn Interessenskonflikte infolge personeller Verflechtungen im Sinne der Verordnung bestehen oder wenn gegen bestehende Rotationspflichten verstoßen wird. ${ }^{129}$ Ratingagenturen würden sich ferner haftbar machen, wenn sie keine geeigneten Verfahren verwenden, um sicherzustellen, dass abgegebene Ratings auf einer gründlichen Analyse aller Informationen basieren, die ihnen zur Verfügung stehen. Diese Methoden und Ratings müssen laufend überprüft werden. ${ }^{130}$ Auch ein Verstoß gegen die umfassenden Offenlegungspflichten gegenüber der ESMA und der Öffentlichkeit kann nun eine Haftung nach sich ziehen. ${ }^{131}$

Als interessensgerecht ist die teilweise Beweislastumkehr zugunsten des Anlegers zu bewerten, wie sie KOM (2011) 747 vorsah. Dieser wird regelmäßig keinen Einblick in das interne Bewertungsverfahren erlangen und steht im Angesicht der nationalen Haftungsansprüche vor nicht lösbaren Beweislastproblemen. ${ }^{132}$ Anerkannte Beweiserleichterungen wie etwa im Arzthaftungsrecht existieren derzeit hier nicht. Deshalb erschien der usprüngliche Vorschlag für die Ausgestaltung der europäischen Haftungsnorm angemessen, dass der Anleger lediglich Tatsachen für das Vorliegen einer Verletzung und des Einflusses der Verletzung auf das Ratingergebnis vorzutragen hat, bei deren Vorliegen vermutet wird, dass eine Zuwiderhandlung der Ratingagentur vorliegt und sich diese auf das Rating ausgewirkt hat. ${ }^{133}$ Diese Beweislastumkehr fiel jedoch einem Kom-

$128 \operatorname{KOM}$ (2011) 747, Art. 35a Abs. 1: »Hat eine Ratingagentur vorsätzlich oder grob fahrlässig eine der in Anhang III aufgeführten Zuwiderhandlungen begangen und hat sich diese auf ein Rating ausgewirkt, auf das ein Anleger beim Erwerb eines bewerteten Instruments vertraut hat, so kann dieser Anleger die Ratingagentur auf Ersatz des ihm entstandenen Schadens verklagen. «(Fn. 43).

129 KOM (2011) 747 Anhang III unter 1. a) bis e) (Fn. 43).

130 KOM (2011) 747 Anhang III unter 1. f) bis h) (Fn. 43).

131 KOM (2011) 747 Anhang III unter 2. und 3 (Fn. 43).

132 So etwa bei $\S 826$ BGB und $\S \S 280$ I, 311 III 2, 241 II BGB.

133 Vgl. Art. 35a IV und Erwägungsgrund 26 KOM (2011) 747 (Fn. 43). 
promiss der Mitgliedstaaten und des Europaparlaments zum Opfer: Zwar wurde in der Diskussion des Vorschlags KOM (2011) 747 eine Einigung dahingehen erzielt, dass Ratingagenturen für vorsätzliche oder grob fahrlässige Fehleinschätzungen haften, die Beweislastumkehr ließ sich jedoch nicht durchsetzen. ${ }^{134}$ Wie entscheidend die Einführung der Beweislastumkehr gewesen wäre, zeigt auch das derzeit anhängige Verfahren gegen Lehman. Der Kläger steht hier vor der Herkulesaufgabe, der Ratingagentur $S \& P$ eine Pflichtverletzung nachzuweisen. ${ }^{135}$ Ohne eine gesetzliche Beweislastumkehr wird dies dem geschädigten Anleger kaum gelingen können. Deshalb war die vorgeschlagene Regelung des Art. 35a nicht nur ein sinnvoller, sondern gar notwendiger Schritt auf dem Weg zur Einführung eines Haftungsregimes. Mit der Streichung der Beweislastumkehr aber bleibt das vermeintlich schärfste Schwert der Regulierungsbemühungen stumpf.

\section{Schaden und Kausalität}

Nach dem Verordnungsvorschlag der Kommission ${ }^{136}$ muss der klagende Anleger darlegen und beweisen, dass ihm durch das fehlerhafte Rating ein ersatzfähiger Schaden entstanden ist. Wie der Schaden zu bestimmen ist, lässt der Vorschlag im Einzelnen offen. In Betracht kommen grundsätzlich zwei Schadenspositionen: Die Geltendmachung der Kursdifferenz (Preiskausalität) ${ }^{137}$ oder die Rückgängigmachung des Anlagegeschäfts ${ }^{138}$.

Im ersteren Fall besteht der Schaden darin, dass ein Finanzprodukt im Vergleich zu einem hypothetisch korrekten Erwerbspreis überteuert erworben oderim umgekehrten Fall - ein tatsächlich zu niedriger Veräußerungspreis erzielt wurde. ${ }^{139}$ Die Darlegung eines Kursschadens dürfte aber nur unter erheblichen Schwierigkeiten gelingen, da dies die Annahme beinhalten würde, dass ein Kurs-

134 FAZ, »Ratingagenturen haften künftig für grob fahrlässige Fehleinschätzungen« v. 29. November 2012, S. 11.

135 Er führt an, dass es »(a)ufgrund des nicht transparenten Ratingverfahrens und der mangelnden Fachkenntnisse insbesondere von privaten Anlegern, (...) den Geschädigten nahezu unmöglich (ist,) zu beweisen, dass die beklagte Rating-Agentur bei der Erstellung des Ratings jeweils ihre Pflichten verletzt hat«. Aufgrund dessen fordert er eine Beweislastumkehr, sodass die Beklagte nachzuweisen hätte, dass ihre Bewertung lege artis erfolgte, Klageschrift, S. 5 f. (Fn. 124).

136 KOM (2011) 747, Erwägungsgrund 26, »(...) Die Beweislast hinsichtlich des Vorliegens eines Schadens und der Ursächlichkeit der Verletzung für den Schaden, die beide eher der Sphäre des Anlegers zuzuordnen sind, sollte hingegen ganz vom Anleger zu tragen sein.« (Fn. 43).

137 Vasella, Haftung von Ratingagenturen, 2011, S. 370.

138 Vgl. auch Bachmann, JZ 2012, $578 \mathrm{ff}$.

139 Vasella (Fn. 137), S. 370. 
sturz das Ausmaß der Fehleinschätzung der Ratingagentur ausdrückt. ${ }^{140}$ Zwar kann sich der geschädigte Anleger eines Gutachters bedienen, die gutachterliche Ermittlung des hypothetisch korrekten Kurses dürfte im Einzelfall aber problematisch und mit erheblichen Risiken für den Anleger verbunden sein. Zum einen entspricht eine Kursdifferenz nicht automatisch dem Grad der Fehlerhaftigkeit eines Ratings, sondern faktoriert noch viele weitere Kriterien wie etwa Markterwartungen mit ein. Zum anderen dürfte die Schwierigkeit auch darin bestehen, den zeitlichen Zusammenhang zwischen dem Rating und dem Zeitpunkt des Schadenseintritts zu beweisen, da der Kurseffekt einer Information auch durch andere Faktoren mit der Zeit überlagert wird. ${ }^{141}$

Alternativ kann der Anleger auch geltend machen, dass das Rating ursächlich für seine Anlageentscheidung war. Der Schaden besteht in diesem Fall in der Differenz des tatsächlichen Werts des Finanzprodukts infolge des Ratings zur Situation, dass der Anleger die Kaufentscheidung nie getroffen hätte. Zu ersetzen ist folglich das negative Interesse oder der $»$ Vertragsabschlussschaden ${ }^{142}$. Der Nachweis kann dem Anleger etwa schon dann gelingen, wenn er belegen kann, dass er generell eine risikoarme Anlagestrategie verfolgt und das zu positiv und damit sicher bewertete Finanzprodukt deshalb in sein Portfolio passte. ${ }^{143}$

\section{Bewertung - keine Haftung für inhaltlich falsche Ratings}

Der Verordnungsvorschlag begründet in erster Linie eine Haftung für Pflichtverstöße gegen formelle Anforderungen der Rating-VO. Solche sind leicht zu definieren und vor allem auch nachprüfbar. Allerdings erscheint fraglich, ob bei Befolgung dieser "Compliance«-ähnlichen Regelungen der Verordnung auch automatisch Fehlbewertungen wie in der Finanzkrise vermieden werden können. Art. 35a umgeht insofern eine Statuierung von Haftungsgründen für inhaltlich »falsche« Ratings.

Ebenso wie bei der Beratung oder der Finanzanalyse ${ }^{144}$ stellt sich das Problem, dass Ratings in gewissem Maße eine nur subjektive Einschätzung über eine zukünftige Entwicklung darstellen. Daher gibt es keine objektiv »richtige« oder »falsche« Bewertung. Ein Rating stellt daher immer eine Gratwanderung zwischen rechtzeitiger und akkurater Information der Marktteilnehmer, um diese vor künftigen negativen Entwicklungen zu schützen, und einem vorschnellen

140 Vasella (Fn. 137), S. 371 ff.

141 Vasella (Fn. 137), S. 372.

142 Barth, Schadensberechnung bei Haftung wegen fehlerhafter Kapitalmarktinformation, 2006, S. 195.

143 Vasella (Fn. 137), S. 375.

144 S. oben D.II.1. 
Downgraden dar, das schnell einen Dominoeffekt auf den Märkten sowie Überreaktion der Markteilnehmer nach sich ziehen kann.

Dieser Ermessensspielraum ist kaum nachprüfbar, unterliegt aber dennoch gewissen Einschränkungen, da die Ratingagenturen ihren Bewertungen auch harte Fakten und Informationen zugrunde legen müssen. Die Daten müssen richtig, vollständig und aktuell sein, um eine zutreffende Bewertung zu erzielen. Eben diese Bewertungsbasis unterliegt der Offenlegung gegenüber der ESMA und ist somit vergleichbar und nachprüfbar. Nach gegenwärtigem Verordnungsvorschlag kann sich eine Ratingagentur aber von der Haftung freizeichnen, wenn sie "geeignete Verfahren beschließt, umsetzt und durchsetzt, um sicherzustellen dass die (...) abgegebenen Ratings (...) auf einer gründlichen Analyse aller Informationen basieren, die ihr zu Verfügung stehen (...) « ${ }^{145}$. Allein die Implementierung eines solchen Kontrollverfahrens garantiert jedoch kaum die »richtige« Bewertung der zugrunde gelegten Informationen. Diese kann wohl nur erfolgen, wenn bezüglich der Informationsbasis selbst bestimmte Pflichten - wie Aktualisierung, Vollständigkeit und Richtigkeit - gesetzlich vorgeschrieben werden. Eine solche Pflicht lässt sich derzeit allenfalls in Anhang III 1. h) KOM (2011) 747 hineinlesen, der besagt, dass ein haftungsbegründender Pflichtverstoß vorliegt, wenn die Ratings und Methoden nicht überwacht oder laufend, "mindestens jedoch einmal jährlich«, überprüft werden. Auch ist zu fordern, dass der Begriff des "geeigneten Verfahrens ${ }^{146}$ genauer zu definieren ist. Der Verordnungsvorschlag überlässt in seiner derzeitigen Fassung den Ratingagenturen erheblichen Interpretationsspielraum, weil der Normgeber dem klassischen Zirkelschluss unterliegt: Wenn ein Rating auf der gründlichen Analyse aller der Agentur zur Verfügung stehenden Informationen basiert, wird das Verfahren für geeignet erachtet. Eine gründliche Analyse setzt aber selbst ein hierfür geeignetes Verfahren voraus.

IV. Sinnvolle Erweiterungen und Präzisierungen der Haftungsgrundlage des Art. 35a

Anhand der Fälle, in denen in jüngerer Zeit Ratingagenturen involviert waren, lässt sich die Haftungsregel des Art. 35a weiter konkretisieren. 
Im Falle Lehman lautet der Vorwurf des Klägers, dass das Rating nicht »methodengerecht unter Auswertung und zutreffender Gewichtung aller vorhandenen Fakten zustande gekommen (sei) ${ }^{148}$. Tatsächlich verteidigte sich die Bank öffentlich insofern, dass der Vertrauensverlust seitens der Anleger in einem Maß eskalierte, das so in seinen Folgen nicht vorhersehbar war. ${ }^{149}$ Gesteht man Ratinganalysten einen Beurteilungsspielraum zu, so lässt sich einem Rating trotzdem ein objektiver Aussagegehalt entnehmen. Hier wurde bis drei Tage vor der Insolvenz die Bewertung "A " durch $S \& P$ aufrecht erhalten. Dies implementiert, dass sich Lehman nicht in finanziellen Schwierigkeiten befand, zumindest aber nicht vor der drohenden Insolvenz stand. Angesichts der sich überschlagenden Meldungen über die drohende Insolvenz von Lehman in den Medien ${ }^{150}$ kann eine derartige Diskrepanz zwischen ex-post-Wirklichkeit und Rating aber nicht mehr dem subjektiven Ermessenspielraum der Analysten im Sinne einer Feinjustierung zugeordnet werden. Vielmehr lag eine Fehl- bzw. Nichtauswertung von öffentlich zugänglichen Informationen vor, in deren Folge es versäumt wurde, das Rating anzupassen und damit den Anlegerkreis zu informieren.

Würde ein vergleichbarer Fall künftig eine Haftung nach Art. 35a auslösen? Es ist davon auszugehen, dass $S \& P$ als eine der führenden Ratingagenturen weltweit grundsätzlich geeignete und standardisierte Verfahrensabläufe und kontrollen zur Abgabe von Ratings implementiert hat. Zudem war im vorliegenden Fall ja gerade nicht das ursprünglich abgegebene Rating mit »A+« umstritten, sondern die Überwachung und Anpassung der Bonitätsnote. Ausweislich des Emissionsprospekts ${ }^{151}$ entsprach das streitgegenständliche Rating dem Stand vom 19.3.2008. Der Zusammenbruch der Lehman-Bank erfolgte etwa sechs Monate später am 15.9.2008. Damit wäre aber $S \& P$ der geplanten Überwachungspflicht in zeitlicher Hinsicht (»laufend, mindestens jedoch einmal jährlich «) ${ }^{152}$ wohl noch vollumfänglich nachgekommen.

147 Zum »know your product« s. ausführlich Möllers/Puhle, JZ 2012, $592 \mathrm{ff}$.

148 Klageschrift, S. 5 (Fn. 124).

149 »We believe the downfall of Lehman reflected escalating fears that led to a loss of confidence - ultimately becoming a real threat to Lehman's viability in a way that fundamental credit analysis could not have anticipated with greater levels of certainty«, CFO v. 24. September 2008, »Rating itself: 'S\&P defends Lehman's 'A'«, http://www.cfo.com/article.cfm/12295579.

150 Vgl. Klageschrift, S. 6 ff. (Fn. 124).

151 Klageschrift, S. 3 (Fn. 124).

152 KOM (2011) 747 Anhang III 1.h) (Fn. 43). 
Anhand dieses Beispiels wird deutlich, dass die im Verordnungsvorschlag gewählten Zeiträume nicht hinreichend ausgestaltet sind. Solche Mindestangaben sind zu starr und werden der Schnelllebigkeit des Finanzmarktes nicht gerecht. Vielmehr erfordern die dynamischen Entwicklungen laufende Überwachungsund Prüfungspflichten, wenn konkrete Anhaltspunkte für Veränderungen der $\mathrm{Pa}$ rameter des Ausfallrisikos gegeben sind, und daraus folgend eine dementsprechende Pflicht zur Anpassung des Ratings. Spätestens wenn in den Medien über die Kreditwürdigkeit eines Unternehmens berichtet wird, muss auch eine Ratingagentur Recherchen aufnehmen. Das hatte $S \& P$ wohl unterlassen oder das Rating zumindest nicht rechtzeitig angepasst. Zieht man hier die Parallele zur Haftung von Anlageberatern ${ }^{153}$, würde sich die Haftung für das Rating im Sinne einer Prognose daraus ergeben, dass ein »A«-Rating aus damaliger ex-ante Sicht im Angesicht der Meldungen in der Presse als völlig unvertretbar erscheint.

\section{Vorschnelles Herunterstufen (Downgraden)}

a) Downgraden bei unzureichender Bewertungsgrundlage

$S \& P$ sorgte auch im Bereich der Länderratings für Aufruhr, als im August 2011 eine höchst umstrittene Herabstufung der Kreditwürdigkeit der USA ${ }^{154}$ und im November desselben Jahres ein irrtümlicher Downgrade der französischen Kreditwürdigkeit ${ }^{155}$ vorgenommen wurde. Im ersten Fall stufte $S \& P$ die USA wegen des steigenden Haushaltsdefizits zum ersten Mal seit 1941 von »AAA « auf "AA+« herab. Dieser Schritt war auch insofern umstritten, weil Moody's gleichzeitig ihr bestehendes »AAA«-Rating trotz der andauernden Haushaltsdebatte aufrecht erhielt. ${ }^{156}$ Das US-Finanzministerium nahm umgehend Stellung und zog das Rating in Zweifel, da $S \& P$ ihrer Analyse falsche Daten zugrunde gelegt und den Schuldenstand deshalb um etwa zwei Billionen US-Dollar zu hoch angesetzt hätte. ${ }^{157}$ Nach Rücksprache mit dem Finanzministerium bestätigte $S \& P$ dies

153 Reinelt (Fn.103), 6 ff.; umfassend jetzt Möllers/Puhle (Fn. 147), 592 ff.

154 »White House challenges S\&P decision«, Wall Street Journal v. 7. August 2011, http://online.wsj.com/article/SB10001424053111903454504576492724028210348.html? mod=rss_whats_news_us_business\&utm_source=twitterfeed\&utm_medium=twitter.

155 »Rating-Panne empört Frankreich«, spiegelonline v. 11. November 2011 (Fn. 3).

156 »United States loses prized AAA credit rating from S\&P«, reuters v. 6. August 2011 (Fn. 4).

157 »United States loses prized AAA credit rating from S\&P«, reuters v. 6. August 2011 (Fn. 4). 
zwar, gab aber an, dass dies die Entscheidung zur Herabstufung in keiner Weise beeinflusst habe. ${ }^{158}$

Auch bei der Bewertung der französischen Kreditwürdigkeit geriet $S \& P$ unter Beschuss: im November 2011 verkündete die Agentur irrtümlich eine Herabstufung Frankreichs von seiner Bestnote "AAA» an. Zwei Stunden, nachdem eine Mitteilung hierüber an Interessenten verschickt wurde, räumte $S \& P$ eine technische Panne ein. Doch waren die Anleger bereits aufgeschreckt und versuchten, ihre französischen Anleihen abzustoßen. ${ }^{159}$

Auch in solchen Fällen nachgewiesener "Pannen « können sich Ratingagenturen von der Haftung des Art. 35a »exkulpieren«, wenn sie nur den Nachweis der Implementierung eines Kontrollverfahrens erbracht haben. Wenn solche Systeme grundsätzlich als Kontrollverfahren geeignet sind, so kann ein Versagen im Einzelfall, wie im Falle Frankreichs, wohl keine Haftung nach sich ziehen. Im Fall der USA stellte $S \& P$ zudem ausdrücklich klar, dass das Informationsdefizit keine Auswirkung auf das Rating habe, sodass hier schon gar kein »unrichtiges« Rating vorliegt, an dem eine Haftung anknüpfen könnte. In concreto dürfte eine solche Behauptung auch nur schwer zu widerlegen sein. Trotz wirtschaftlich betrachtet enormer Auswirkungen, handelt es sich bei der Herabstufung von "AAA" zu "AA+" und im Vergleich zum Rating des Marktkonkurrenten Moody's ("AAA») technisch nur um einen graduellen Unterschied, der auf unterschiedlichen Modellen der Bewertung basieren kann und der letztlich auch dem Ermessenspielraum der Ratingagentur zugerechnet werden muss.

b) Verpflichtung zur Erstellung von sog. Rating-Outlooks

Vor der tatsächlichen Herabstufung der Kreditwürdigkeit bedienen sich Ratingagenturen aber häufig Ankündigungen ${ }^{160}$, die zum einen die Anleger sensibilisieren sollen, zum anderen aber auch der gerateten Partei Gelegenheit zur Stellungnahme sowie gegebenenfalls zum Hinweis auf Fehler in der Bewertung geben. Im Fall von Unternehmensratings schreibt die Rating-VO ${ }^{161}$ sogar vor, dass die Agentur das bewertete Unternehmen mindestens 12 Stunden vor der Veröffentlichung des Ratings und über die wichtigsten Gründe zu informieren hat, um dem Unternehmen die Möglichkeit zu geben, auf sachliche Fehler der Agentur hin-

158 »United States loses prized AAA credit rating from S\&P«, reuters v. 6. August 2011 (Fn. 4).

159 »Rating-Panne empört Frankreich«, spiegelonline v. 11. November 2011 (Fn. 3).

160 So etwa die Stellungnahme von Fitch zu Konsequenzen für die Kreditwürdigkeit der Länder der Eurozone im Falle eines Austritts Griechenlands, »Fitch: Bei GriechenlandAustritt droht Downgrade für gesamte Eurozone«, Deutsche Mittelstands Nachrichten v. 12. Mai 2012, http://www.deutsche-mittelstands-nachrichten.de/2012/05/42536/

161 Anhang I Abschnitt D Teil 1 Nr. 3 Rating-VO (Fn. 21). 
zuweisen. Im neuen Verordnungsvorschlag ${ }^{162}$ soll diese Frist auf »einen vollen Arbeitstag« vor Veröffentlichung verlängert werden.

Um dem Interessenskonflikt zwischen Anlegerschaft und Ratingobjekt gerechter zu werden, bedienen sich die Agenturen auch sog. »Rating-Outlooks «. Dies sind Prognosen, ob sich ein Rating auf längere Sicht positiv, stabil oder negativ entwickeln wird. ${ }^{163}$ Der neue Verordnungsvorschlag weitet die für Ratings geltenden Vorschriften auch auf Rating-Outlooks aus ${ }^{164}$ und stellt eigene Anforderungen an die Prognosen, wie etwa die Angabe eines Zeithorizonts, bis zu dem eine Änderung des Ratings zu erwarten ist. ${ }^{165}$ Angesichts des enormen wirtschaftlichen Schadens, den eine Herabstufung nach sich ziehen kann, ist die Verwendung von Ankündigungen und Rating-Outlooks durchaus zu begrüßen und es zeigt sich, dass auch die Agenturen selbst um einen Interessensausgleich bemüht sind.

3. Inhaltlich nicht vertretbares Rating

a) Rating-Shopping

Während es früher als Tabubruch galt, stellen heute zunehmend Ratingagenturen die Bewertungen ihrer Konkurrenten öffentlich in Frage. So kritisierte Fitch im Frühjahr 2012 die Bonitätsnoten, die von $S \& P$ in einem 730 Mio. Dollar schweren Hypotheken-Bond-Deal der Credit Suisse Gruppe vergeben wurde, als zu gut. Nachdem Fitch um eine Einschätzung der Transaktion gebeten worden war, wurde der Auftrag letztlich $S \& P$ erteilt. Die Nichtbeauftragung von Fitch sei auf »der konservativeren Krediteinschätzung der Agentur« zurückzuführen, so Fitch in einem Statement ${ }^{166}$. Ein Sprecher von Credit Suisse bezeichnete die Einholung mehrerer Einschätzungen als einen üblichen Vorgang und $S \& P$ reagierte lediglich mit der Äußerung, dass der Markt von unterschiedlichen Meinungen profitiere. $^{167}$

Auch Moody's kritisierte $S \& P$ kürzlich in anderer Angelegenheit. Konkret sei das Rating für die Exeter Finance Corp. im Zuge einer Kreditverbriefung zu po-

162 KOM (2011) 747 Anhang I Nr. 4 d) (Fn. 43).

163 Arntz, BKR 2012, 89.

164 Vgl. KOM 747 (2011), S. 8; Erwägungsgrund 4 (Fn. 43).

165 KOM (2011) 747 Anhang I, 4. b) iii) KOM (2011) 747 (Fn. 43).

166 »Fitch Blasts S\&P Rating on Credit Suisse Mortgage Bonds«, Bloomberg v. 30. März 2012, http://www.bloomberg.com/news/2012-03-30/fitch-blasts-s-p-ratings-on-creditsuisse-mortgage-securities.html.

167 »Fitch Ditched in Bond Dispute«, Wall Street Journal v. 3. April 2012, http://online.wsj. com/article/SB $10001424052702304750404577322122497596882 . \mathrm{html}$. 
sitiv ausgefallen. ${ }^{168} S \& P$ habe die Anleihen mit "AA " bewertet, obwohl die Exeter Finance Corp. bisher nicht bewertet worden sei, nur geringe Erfahrungswerte bestünden und nur eine kurze Asset-Performance-History vorhanden sei. ${ }^{169}$ Moody's bezeichnete das Rating gar als munangemessen $\ll .{ }^{170}$ Ähnliche Vorfälle liegen schon etwas länger zurück: Im Februar 2011 äußerten Moody's und S\&P gemeinsam Zweifel am "AAA«-Rating durch Fitch im Zuge einer hypothekarisch gesicherten Wertpapiertransaktion ${ }^{171}$ und im Jahr 2010 hatte S\&P Moody's Top-Noten für Bauspar-Anleihen in Zweifel gezogen. ${ }^{172}$

Der verschärfte Umgangston ist auch darauf zurückzuführen, dass Moody's Marktanteile im Segment "structured-finance business« auf Kosten von S\&P gewinnen konnte. ${ }^{173}$ Dabei wird ein offenes Geheimnis der Branche öffentlich thematisiert: Emittenten beauftragen diejenige Agentur, die das beste Rating verspricht (sog. "Rating-Shopping«). Konkret lautet der Vorwurf, dass die Agenturen ihre Standards senken, um bessere Bonitätsurteile abgeben zu können und damit aber gleichzeitig die Risiken nicht mehr korrekt widerspiegeln. Insbesondere kleinere Agenturen bedienten sich dieser Methode im Kampf um Marktanteile. $^{174}$

b)

Ermessensspielraum bei der Erstellung eines Ratings

Auch in den vorstehend geschilderten Fällen ist die Annahme eines Haftungsfalles mehr als problematisch. Zwar können die Ratings verschiedener Agenturen als Vergleichsmaßstab herangezogen werden, aber auch diese beanspruchen das Wesen einer subjektiven Prognoseentscheidung für sich. Somit kann das Bonitätsurteil oder die Einschätzung einer Ratingagentur nicht als objektiver Grad-

168 "Moody's attacks rivals on debt risks«, Financial Times v. 16. März 2012, http://www.ft.com/cms/s/0/0013acc8-6ee4-1 lel-afb8-00144feab49a.html\#axzzlw3a9FD $3 a$.

169 "Securization Risk Rising as Standards Ease, Moody’s says«, Bloomberg v. 15. März 2012, http://www.bloomberg.com/news/2012-03-15/securitization-risk-rising-as-standards-ease-moody-s-says-1-.html.

170 »inappropriate«, «Securization Risk Rising as Standards Ease, Moody’s says«, Bloomberg v. 15. März 2012 (Fn. 169).

171 »Fitch Ditched in Bond Dispute«, Wall Street Journal v. 3. April 2012 (Fn. 167).

172 "Fitch Blasts S\&P Rating on Credit Suisse Mortgage Bonds«, Bloomberg v. 30. März 2012 (Fn. 166).

173 »Securization Risk Rising as Standards Ease, Moody’s says«, Bloomberg v. 15. März 2012 (Fn. 169).

174 So Fitch's Chief Executive Paul Taylor, "Fitch Ditched in Bond Dispute«, Wall Street Journal v. 3. April 2012, (Fn. 167): Interessanterweise war Fitch in den 1990er Jahren, als sich die Agentur zu etablieren versuchte, auch den Vorwürfen des rating-shoppings ausgesetzt, was Taylor nun aber weit von sich weist. 
messer für die Richtigkeit des Ratings einer anderen Agentur dienen. Zudem fällt auf, dass in den geschilderten Fällen die Kritik übende Ratingagentur überhaupt kein Rating abgegeben hat, sondern lediglich die Bewertung der Konkurrenz in Zweifel zog. ${ }^{175}$ Lediglich im Fall des Ratings der Credit Suisse Group gab Fitch eine fundierte eigene Einschätzung ab. ${ }^{176}$

Die Informationsgrundlage der Äußerung der konkurrierenden und nicht beauftragten Agenturen steht somit hinter der der tatsächlich bewertenden Agentur zurück. Abweichende Einschätzungen können sich hieraus ebenso ergeben wie aus der Verwendung unterschiedlicher Methoden und Modelle. Auch drängt sich der Verdacht auf, dass ein wenig "Säbelrasseln« eben zum Geschäft gehört. Solange Ratings verschiedener Agenturen nicht um mehrere Stufen voneinander abweichen und auf der gleichen Informationsbasis beruhen, müssen graduelle Unterschiede auch hier wieder dem Ermessenspielraum der Analysten zugestanden werden. Schließlich wäre ein Schaden wohl nur schwer nachweisbar.

\section{E. Schlussbetrachtung}

\section{Steigerung der Transparenz und Qualität}

Die Regulierungsbemühungen dauern an. Es ist unbestritten, dass die derzeitigen Regularien noch nicht ausreichen, um die während der Finanzkrise zutage getretene Problematik um die Ratingagenturen zu lösen. Auch der neue Vorschlag der Kommission zur Änderung der Rating-VO erkennt dies an und dringt auf weitere Maßnahmen. Nicht jeder dieser ehrgeizigen Plänc hielt jedoch der Diskussion mit den Mitgliedstaaten und dem Europaparlament stand, sodass an entscheidenden Stellen - wie dargestellt - der Rotstift angesetzt wurde. Jedoch ist im Ergebnis das umfassende Maßnahmenpaket der Europäischen Union zur Steigerung der Transparenz als ein erster wichtiger Schritt zur Qualitätskontrolle der Ratingagenturen zu bewerten. Insbesondere die Begründungspflicht ${ }^{177}$, die eine Offenlegung der herangezogenen Quellen und angewandten Methoden vorschreibt, ermöglicht es, das Rating auf seine Qualität und Aussagekraft nachzuvollziehen.

Die Durchsetzung der Offenlegungspflichten muss aber seitens der ESMA mit aller Konsequenz verfolgt werden. So wurden in einer ersten Untersuchung erhebliche Mängel im Bereich Transparenz, Offenlegung, Systemkontrolle und der Implementierung von internen Kontrollmechanismen bei allen geprüften Ratin-

175 »Securization Risk Rising as Standards Ease, Moody’s says«, Bloomberg v. 15. März 2012 (Fn. 169); »Moody's attacks rivals on debt risks«, Financial Times v. 16. März 2012 (Fn. 168).

176 »Fitch Ditched in Bond Dispute«, Wall Street Journal v. 3. April 2012 (Fn. 167).

177 Art. 8 Abs. 2 in Verbindung mit Anhang I Abschnitt D Teil I Rating-VO (Fn. 21). 
gagenturen aufgedeckt. ${ }^{178}$ Die Aufsichtsbehörde hat weitere Untersuchungen angekündigt.

\section{Durchbrechung des Oligopols}

Nach wie vor besteht eine zu große Abhängigkeit von Ratings der marktführenden Ratingagenturen. Positiv ist der Vorschlag der Kommission zu zwei obligatorischen Ratings. Dass er jedoch nicht weit genug geht, beweisen auch die jüngsten Entwicklungen um die von Roland Berger konzipierte europäische Ratingagentur. ${ }^{179}$ Schon jetzt scheinen potentielle Investoren Zweifel an der zukünftigen Marktakzeptanz der Agentur zu hegen. Von Seiten der Politik sei die Verbesserung des Wettbewerbs zwar erwünscht, letztlich aber »eine Frage der Marktteilnehmer, nicht des Staates ${ }^{180}{ }^{180}$. Der Gesetzgeber scheut aus bereits dargelegten Gründen einen direkten Eingriff ins Ratingwesen, verkennt aber die Alternativen zur Errichtung einer hoheitlich getragenen europäischen Ratingagentur. Durch den gesetzlichen Zwang zu einem (zweiten) europäischen Rating könnten "zwei Fliegen mit einer Klappe« geschlagen werden. Nicht nur würde die oligopolistische Struktur des Marktes durchbrochen, sondern der Gesetzgeber könnte vielmehr den bisherigen kleinen europäischen oder neuen Ratingagenturen wie Roland Berger eine entscheidende Starthilfe verschaffen. Mit einem solchen Formulierungsvorschlag könnte der Gesetzgeber den europäischen Agenturen den Zugang auf den Ratingmarkt ebnen und gleichzeitig für verbesserten Wettbewerb und einer damit einhergehenden Qualitätssicherung der Ratings sorgen. Auch die Wiederauflage der Idee einer europäischen Ratingagentur bleibt mit Spannung abzuwarten.

\section{Gesetzliche Verweisungen auf Ratings}

Auch die Abhängigkeit von Ratings durch gesetzliche Verweise besteht fort. Zwar hat im Zuge der Finanzkrise ein Umdenken stattgefunden, Basel III will aber nicht gänzlich auf Ratings verzichten, sondern deren Einfluss lediglich reduzieren. Hier ist eine konsequentere Umsetzung gefragt, um die bestehenden Strukturen tatsächlich aufzubrechen.

178 ESMA Press Release v. 22. März 2012, http://www.esma.europa.eu/system/files/2012220.pdif.

179 "Europäische Ratingagentur von Roland Berger auf der Kippe«, FTD v. 16. April 2012.

180 Sprecher des Finanzministeriums Kotthaus, FAZ v. 16. April 2012, S. 11. 
Obwohl die zivilrechtliche Haftung von Ratingagenturen vehement gefordert wird $^{181}$, mehren sich auch kritische Stimmen: So wird befürchtet, dass Ratingagenturen zu übergroßer Vorsicht neigen könnten und die abgegebenen Bewertungen wiederum an Qualität einbüßen würden. ${ }^{182}$ Sollten sich Ratingagenturen in Bezug auf komplexe Finanzprodukte in Zukunft zu großen Haftungsfolgen ausgesetzt sehen, so wird sogar ein »Ratingstop» für diese Produkte und damit eine Stilllegung des Marktes prophezeit ${ }^{183}$, wie in den USA bezüglich des ABSMarktes geschehen. ${ }^{184}$ Auch wird ein Haftungsregime wohl als eine Art psychologische Barriere für neue, auf den Markt strebende Agenturen bezeichnet. ${ }^{18}$

Durchaus wahrscheinlich ist, dass die Ratingagenturen das Haftungsrisiko durch Preiserhöhungen auf Investoren und Emittenten abwälzen werden. So kündigte $S \& P$ schon jetzt Preisanstiege an, die sich teilweise auf mehr als das doppelte der bisherigen Kosten belaufen sollen. In einem offenen Brief ${ }^{186}$ an $S \& P$ wandten sich 12 deutsche Großkonzerne (u. A. Bayer, E.ON, Daimler und Siemens) gegen die Preisanstiege und bezeichneten sie als "schlichtweg inakzeptabel«. Da eine Kartellbildung im Raum stehe, behielten sich die Unternehmen ausdrücklich weitere Schritte gegen die Agentur vor. Offiziell wurden die Preisanstiege von Seiten von $S \& P$ nicht begründet; vielmehr wurde eine Stellungnahme mit Verweis auf vertrauliche Kundenbeziehungen verweigert. ${ }^{187}$ Diese Geschehnisse sind aber wohl auch vor dem Hintergrund der neuen delegierten Verordnung ${ }^{188}$ der EU zu sehen: künftig werden die Kosten der Beaufsichtigung der Ratingagenturen durch die ESMA von den Ratingagenturen selbst zu tragen sein.

Die Einführung eines Haftungssystems würde für die Ratingagenturen den Anreiz zur Selbstkontrolle deutlich steigern. Dies wiederum würde sich positiv

181 So etwa im Bundestag, Antrag der CDU/CSU und FDP: Ratingagenturen besser kontrollieren, BT-Drucks. $17 / 7638$ v. 9. November 2011, S. 4; Bundespräsident Wulff in Handelsblatt v. 10. Juli 2011, http://www.handelsblatt.com/politik/deutschland/bundespraesident-wulff-will-ratingagenturen-finanziell-haften-lassen/4378148.html.

182 Bremer, DAI 2012, $32 \mathrm{f}$.

183 Harper (Fn. 111), 1964.

184 Vgl. Harper (Fn. 111), 1926, 1964.

185 Bremer (Fn. 182), $32 \mathrm{f}$.

186 »Deutsche Großkonzerne wehren sich gegen Preiserhöhungen von S\&P«, reuters v. 23. April 2012 (Fn. 1).

187 »Deutsche Großkonzerne wehren sich gegen Preiserhöhungen von S\&P«, reuters v. 23. April 2012, (Fn. 1).

188 Delegierte Verordnung (EU) Nr. 272/2012 der Kommission v. 7. Februar 2012 zur Ergänzung der Verordnung (EG) Nr. 1060/2009 des Europäischen Parlaments und des Rates in Bezug auf die Gebühren, die den Ratingagenturen von der Europäischen Wertpapier- und Marktaufsichtsbehörde in Rechnung gestellt werden, Abl. Nr. L 90, 6. 
in der Qualität der Ratings niederschlagen ${ }^{189}$. Der Vorschlag einer Haftung ist damit positiv zu bewerten.

189 Zur präventiv-disziplinierenden Wirkung s. Arntz (Fn. 163), 89. 